\title{
Theory of the Coherence of Topological Lasers
}

\author{
Ivan Amelio® and Iacopo Carusotto \\ INO-CNR BEC Center and Dipartimento di Fisica, Università di Trento, 38123 Povo, Italy
}

(Received 28 February 2020; revised 26 August 2020; accepted 22 October 2020; published 24 December 2020)

\begin{abstract}
We present a theoretical study of the temporal and spatial coherence properties of a topological laser device built by including saturable gain on the edge sites of a Harper-Hofstadter lattice for photons. For small enough lattices, the Bogoliubov analysis applies, and the coherence time is almost determined by the total number of photons in the device in agreement with the standard Schawlow-Townes phase diffusion. In larger lattices, looking at the lasing edge mode in the comoving frame of its chiral motion, the spatiotemporal correlations of long-wavelength fluctuations display a Kardar-Parisi-Zhang (KPZ) scaling. Still, at very long times, when the finite size of the device starts to matter, the functional form of the temporal decay of coherence changes from the KPZ stretched exponential to a Schawlow-Townes-like exponential, while the nonlinear dynamics of KPZ fluctuations remains visible as a broadened linewidth as compared to the Bogoliubov-Schawlow-Townes prediction. While we establish the above behaviors also for nontopological 1D laser arrays, the crucial role of topology in protecting the coherence from static disorder is finally highlighted: Our numerical calculations suggest the dramatically reinforced coherence properties of topological lasers compared to corresponding nontopological devices. These results open exciting possibilities for both fundamental studies of nonequilibrium statistical mechanics and concrete applications to laser devices.
\end{abstract}

DOI: $10.1103 /$ PhysRevX.10.041060

\section{INTRODUCTION}

The laser is one of the most fundamental tools in modern science [1,2]. Its defining feature is the emission of radiation with unprecedented long coherence length and time. This feature makes laser sources essential ingredients in a wide range of applications and justifies the continuous theoretical and technological research of new devices. Also, from a fundamental science perspective, the physical mechanisms underlying laser oscillation represent an archetypical model at the crossroad of nonlinear physics, nonequilibrium statistical mechanics, and quantum optics [3-6].

Recent years have witnessed an explosion of the field of topological photonics $[7,8]$. Following the ground-breaking works [9], experiments have initially focused on demonstrating the topologically protected chiral propagation of light along the edges of passive photonic lattices displaying topologically nontrivial band structures in a variety of different material platforms and frequency regions, from microwaves in two-dimensional photonic crystal structures

Published by the American Physical Society under the terms of the Creative Commons Attribution 4.0 International license. Further distribution of this work must maintain attribution to the author(s) and the published article's title, journal citation, and DOI.
Subject Areas: Condensed Matter Physics

embedding time-reversal-breaking magneto-optical elements [10] to optical light propagating along an array of evanescently coupled helical waveguides laser written in glass [11], to arrays of coupled ring microcavities in a silicon photonics platform [12].

In the past years, in addition to nonlinear topological photonics effects [13-15], major attention has been devoted to laser operation in topological edge modes [16-26]. Such topological lasing is of greatest interest in 2D lattice models: The topologically protected chiral motion of their 1D edge modes suggests the possibility to efficiently phase lock the field at different sites, so to increase the total emission power by distributing gain on a large number of sites and at the same time maintaining a stable single-mode emission of the whole system. So far, realizations of such devices include symmetry-protected spin-Hall states in microcavity arrays [16-19], photonic crystals breaking time reversal via the magneto-optic effect [20], and more recently a valley Hall system with a quantum cascade active medium [27]. Topological lasing was also theoretically studied in quasiperiodic 1D photonic crystals [21] and experimentally observed on the $0 \mathrm{D}$ edge states of topological 1D arrays [22-25]. Very recent developments include the realization of topological bulk lasing in patterned nanodisk arrays [28] and a proposal to obtain stable mode-locked laser operation in a Floquet system with one real and one synthetic dimension [29]. 
Pioneering theoretical studies focus on the classical properties of the emission at the mean-field level $[18,30]$, highlighting the promise of topological lasers with respect to the power slope efficiency (that is, the derivative of the emitted laser intensity with respect to the input pump power) and to the robustness of the single-mode monochromatic emission in the presence of static disorder. A detailed study of the temporal and spatial coherence properties of the laser emission including the effect of noise due, e.g., to spontaneous emission is instead still missing. The characterization of the ultimate limitations to the coherence properties is a key element in view of technological applications of topological lasers and is the main topic of this work.

As it was first theoretically understood by Schawlow and Townes [31], spontaneous emission events determine an inescapable diffusion of the phase of the macroscopic electromagnetic field, which results in an exponential decay of the temporal coherence function and in a corresponding spectral linewidth. In this analysis, the spatial extension of the laser field is irrelevant, and the dynamics is effectively restricted to the phase of a single complex number. In analogy with this ultimate Schawlow-Townes linewidth, a natural question is to investigate the ultimate limitations to the coherence of a spatially extended topological laser device. Here, the evolution of the field involves a complex spatiotemporal dynamics over many coupled sites.

The goal of this work is to make use of nonequilibrium statistical mechanics concepts to investigate how spatial fluctuations affect temporal coherence in topological laser devices. From the very beginning, topological lasing was proposed as a very promising strategy in order to achieve phase locking in arrays of many semiconductor lasers and make them operate as a single laser [16], which is a fundamental challenge in laser science [32-34]. Setting up a quantitative description of such devices is a highly challenging theoretical task, as it involves a strongly nonlinear dynamics and is sensitive to the microscopic details of the photonic structure and of the gain material. Here, we make an important step in the direction by analyzing in full detail a simplest model of topological laser. In particular, we focus on a tight-binding HarperHofstadter model with no polarization degrees of freedom, and we restrict to the simplest scenario of so-called class-A lasers [35,36]. In such devices, light amplification is provided by a saturable gain medium with a temporally instantaneous response, and the carrier dynamics in the gain medium and the intensity dependence of the real part of the refractive index are neglected.

These simplifying assumptions are typically far from being fulfilled in topological laser devices based on semiconductor laser technology $[17,19,22,23,27]$. Nonetheless, as long as the topological laser operation remains single mode as in the experiments $[18,20]$, we expect that the specific features of the actual device will give quantitative modifications to the coherence length and time (for instance, in terms of linewidth broadening factors à la Henry [35,37]) but will not affect the qualitative structure of the spatiotemporal correlation functions. These are, in fact, instead determined by the universal properties of the underlying nonequilibrium dynamics. Going beyond these approximations would require an $a b$ initio treatment of laser oscillation, starting from Maxwell equations for the electromagnetic field and a microscopic description of the gain medium $[35,38]$. Such calculations are the subject of future work and are of great interest also to precisely characterize the role of topology in suppressing dynamical instabilities [36,39] and ruling out multimode behaviors.

The starting point of our study are the recent theoretical works [40-45] which anticipate that (for small-intensity fluctuations) the long-wavelength dynamics of the phase in an array of coupled laser resonators is captured by the celebrated Kardar-Parisi-Zhang (KPZ) model of nonequilibrium statistical mechanics [46]. Since topological laser operation occurs into a one-dimensional edge mode, it is natural to search for a KPZ dynamics also in this case. As a first result of our work, we numerically show that this anticipation is indeed the case once the phase dynamics is studied in the reference frame moving at the group velocity of the chiral mode. In particular, we highlight regimes where the spatiotemporal correlation functions accurately satisfy the KPZ scaling with no dramatic renormalization of the nonlinear coupling.

While strictly speaking the KPZ scaling is found in spatially infinite systems, the situation is completely different for the behavior of realistic devices which necessarily have a finite spatial size. This study requires an explicit analysis of finite-size effects beyond what is normally considered in the nonequilibrium statistical mechanics literature. For relatively small lattices, a linearized Bogoliubov analysis is accurate and yields a transverse Petermann factor $[47,48]$ close to unity, demonstrating the effectiveness of topological mode guiding. In agreement with the standard Schawlow-Townes picture, the coherence then decays according to an exponential law with a coherence time determined by the ratio of the total number of photons in the device over the noise rate. In large (but still finite) systems, a remarkably different behavior is found: At very long times, the coherence still decays exponentially à la Schawlow-Townes and does not show the characteristic KPZ scaling, but the evolution of the phase keeps memory of the underlying KPZ dynamics, and the phase diffusion rate gets strongly reinforced by the intrinsic nonlinearity of the KPZ model. These findings apply to generic nonequilibrium phase transitions where a $\mathrm{U}(1)$ symmetry is spontaneously broken such as BoseEinstein condensation of exciton polaritons [49,50], complementing the previous KPZ studies [40-45].

As a final point of our work, we demonstrate that the coherence of a topological laser is very weakly affected by 
a static disorder. Coherence is lost only when the strength of disorder is on the order of the topological gap, that is far higher than what is needed to spoil the coherence of a nontopological array. For intermediate values of the disorder strength, we numerically find a striking enhancement of the coherence time almost up to the Schawlow-Townes value, which can be explained in terms of an effective suppression of the KPZ nonlinear effects by disorder.

The structure of the article is the following. In Sec. II, we focus on finite 1D laser chains, and, after inspecting the crossover from the Kardar-Parisi-Zhang scaling to a Schawlow-Townes-like phase diffusion at very long times, we illustrate the significant reduction of the coherence time from the Bogoliubov-Schawlow-Townes prediction due to the nonlinear dynamics of the spatial fluctuations of the phase. In Sec. III, we first review the basic concepts of topological laser operation into the chiral edge mode of a 2D Harper-Hofstadter lattice. We then investigate the emission spectrum and the one-dimensional character of the phase fluctuations. In particular, we probe the universal KPZ scaling of long-wavelength fluctuations at intermediate times, and we compute the linewidth of the laser emission at very long times. In Sec. IV, we demonstrate the robustness of the coherence of the topological laser emission in the presence of a static disorder. A critical discussion of the applicability of our results to specific topological laser devices is given in Sec. V. Conclusions and perspectives are finally drawn in Sec. VI.

\section{ONE-DIMENSIONAL ARRAY OF LASER RESONATORS}

In this section, we make use of a semiclassical approach based on stochastic differential equations to characterize the coherence properties of a simple model of spatially extended laser formed by a one-dimensional array of single-mode resonators. While a sizable literature has investigated the behavior of long-wavelength fluctuations in terms of the KPZ equation [40-45], not much attention has been paid to the temporal dependence of the equalspace correlation function of spatially finite systems, which is one of the experimentally most relevant quantities for lasers. To facilitate the reader, we first set the stage by reviewing the Schawlow-Townes linewidth for single-laser resonators, and then we move up to the case of interest of a finite array of coupled resonators.

\section{A. Single resonator linewidth}

The basic features of laser operation in a single-mode resonator, namely, stimulated amplification, the spontaneous breaking of the U(1) symmetry of the field, and the stabilization of the emission intensity by gain saturation, are all captured by the stochastic differential equation $[4,37,50-52]$

$$
i \partial_{t} \psi=\frac{i}{2}\left[\frac{P}{1+n / n_{S}}-\gamma\right] \psi+\sqrt{2 D} \xi
$$

for the single complex variable $\psi$ describing the amplitude of the electromagnetic field in the resonator, $n=|\psi|^{2}$ is the field intensity, $\gamma$ is the loss rate of the "cold" resonator, and $P$ is the amplification rate induced by the (unsaturated) gain. As already stressed in the introduction, a key assumption of our work is that the gain medium has an instantaneous response to the light intensity $n$, with a gain saturation coefficient $n_{S}$. Even though existing realizations of topological lasers [17,19,20,22,23,27] do not fall in the category of such class-A lasers, this simplifying assumption facilitates our task of getting insight in the universal properties of topological laser devices. As usually done in the semiclassical theory of lasers, the stochastic term $\xi$ accounts for noise due, e.g., to spontaneous emission. It is taken in the form of a spatiotemporally white noise of unit variance $\left\langle\xi^{*}(t) \xi\left(t^{\prime}\right)\right\rangle=\delta\left(t-t^{\prime}\right)$ rescaled to have a diffusion coefficient $D$.

Equation (1) is invariant under a U(1) symmetry describing the rotation of the phase of the field $\psi$. The steady-state field $\psi_{0}$ is zero below the lasing threshold $P<P_{\text {th }}=\gamma$. Above the threshold $P>P_{\text {th }}$, the steady-state field $\psi_{0}$ spontaneously breaks the symmetry by choosing a specific phase, while the steady-state intensity is fixed to $n_{0}=\left|\psi_{0}\right|^{2}=n_{S}\left[\left(P / P_{\mathrm{th}}\right)-1\right]$. For $P=2 P_{\mathrm{th}}$, one has the very transparent expression $n_{0}=n_{S}$.

To deal analytically with Eq. (1), it is convenient to resort to the modulus-phase formalism: Writing the field as $\psi=\sqrt{n} e^{i \phi}$, one has

$$
\begin{gathered}
\partial_{t} \phi=\sqrt{\frac{D}{n}} \xi_{1}, \\
\partial_{t} n=\left[\frac{P}{1+n / n_{S}}-\gamma\right] n+2 \sqrt{n D} \xi_{2}
\end{gathered}
$$

in terms of the two real and independent noises $\left\langle\xi_{l}(t) \xi_{l^{\prime}}\left(t^{\prime}\right)\right\rangle=\delta_{l, l^{\prime}} \delta\left(t-t^{\prime}\right), l, l^{\prime}=1$, 2. For small enough perturbations, the intensity relaxes to $n_{0}$ at a rate $\Gamma=[\gamma(P-\gamma) / P]$. On the other hand, as a direct consequence of the U(1) symmetry of the model, the dynamics of the phase is a diffusion with no restoring force.

Assuming noise is small enough to cause minor perturbations to the intensity, phase differences follow a Gaussian distribution, so within the so-called cumulant approximation [40], the autocorrelation of the field reads

$$
g^{(1)}(t)=\left\langle\psi^{*}(t) \psi(0)\right\rangle \simeq n_{0} e^{-(1 / 2)\left\langle[\phi(t)-\phi(0)]^{2}\right\rangle} .
$$

As a consequence, the usual Brownian motion scaling entails that the decay of coherence is described by an exponential law 


$$
g^{(1)}(t) \simeq n_{0} e^{-\left(\gamma_{\mathrm{ST}, 1} / 2\right)|t|}
$$

corresponding to a Lorentzian power spectral density. The coherence decay rate

$$
\gamma_{\mathrm{ST}, 1}=\frac{1}{\tau_{\mathrm{ST}, 1}}=\frac{D}{n_{0}}
$$

is the celebrated Schawlow-Townes linewidth [31,37]. The crucial feature of this formula for the linewidth is the steady-state intensity $n_{0}$ appearing at the denominator, which has the following physical interpretation: The more photons are present in the resonator, the less the phase of the field is perturbed when an additional photon with a random phase is emitted into the mode by a spontaneous emission process $[1,2,52]$.

\section{B. Extended lasers and KPZ equation}

In analogy to the Schawlow-Townes linewidth of lasing in a single-mode resonator, the naive intuition for an array of coupled resonators is that the laser linewidth is inversely proportional to the total number of photons that are present within some correlation length. For small enough lattices, all resonators oscillate in phase, and, for a given number of photons per resonator $n_{0}$, the coherence time is proportional to the length of the array. On the other hand, for large enough systems, it is known that the physics is described by the KPZ equation, which leads to different scaling laws. The main goal of this section is to connect these two points of view into a unified perspective.

To attack this question in a quantitative way, we make use of a generalization of Eq. (1) to a one-dimensional array of $N_{x}$ coupled laser resonators $x=1, \ldots, N_{x}$ :

$i \partial_{t} \psi_{x}=-J \psi_{x+1}-J \psi_{x-1}+\frac{i}{2}\left[\frac{P}{1+\frac{n_{x}}{n_{S}}}-\gamma\right] \psi_{x}+\sqrt{2 D} \xi_{x}$,

with periodic boundary conditions and independent noises $\left\langle\xi_{x}^{*}(t) \xi_{x^{\prime}}\left(t^{\prime}\right)\right\rangle=\delta_{x x^{\prime}} \delta\left(t-t^{\prime}\right)$. In this work, we always consider weak noise, that is, $D$ small enough (corresponding to density fluctuations of the order of $15 \%$ ) to avoid the generation of topological defects, so that the winding number over the chain is conserved during the dynamics.

For large $J$ and calling $a$ the distance between neighboring resonators, one can interpret Eq. (7) as a discrete version of a continuous field of mass $m=\left(1 / 2 J a^{2}\right)$. The corresponding continuous equation is the complex Ginzburg-Landau equation [53]. Assuming fast relaxation of the intensity fluctuations, one can focus on the dynamics of the phase (the derivation is reviewed in Supplemental Material [54]), which is described by the KuramotoSivashinsky equation (KSE)

$$
\partial_{t} \phi=\frac{1}{2 m}\left[-\frac{\Gamma^{-1}}{2 m} \partial_{x}^{4} \phi-\left(\partial_{x} \phi\right)^{2}\right]+\sqrt{\frac{D}{n_{0}}} \xi_{1} .
$$

Here, $\phi$ is the unwound phase living on the real axis and not the compact one restricted to $[0,2 \pi]$.

The characteristic scales of the system as a function of the microscopic parameters are reported in Ref. [40]:

$$
\begin{aligned}
l^{*} & =\left[\frac{J^{4}}{\Gamma^{3} D n_{0}^{-1}}\right]^{1 / 7}, \quad t^{*}=\left[\frac{J^{2}}{\Gamma^{5}\left(D n_{0}^{-1}\right)^{4}}\right]^{1 / 7}, \\
\phi^{*} & =\left[\frac{\left(D n_{0}^{-1}\right)^{2}}{J \Gamma}\right]^{1 / 7} .
\end{aligned}
$$

Measuring space, time, and (unwound) phase in terms of $l^{*}$, $t^{*}$, and $\phi^{*}$, respectively (and after sending $\phi \rightarrow-\phi$ ), the adimensional KSE reads

$$
\partial_{\tilde{t}} \tilde{\phi}=-\partial_{\tilde{x}}^{4} \tilde{\phi}+\left(\partial_{\tilde{x}} \tilde{\phi}\right)^{2}+\tilde{\xi} \text {. }
$$

However, since other scales can be relevant for the 2D topological laser discussed below, in most of the paper we use the physical (without tilde) space, time, and phase variables. A rescaling is proposed in order to study KPZ features in Sec. III D.

The renormalization group analysis shows that at long distances and times the KSE flows to the KPZ universality class [55]. The KPZ equation reads

$$
\partial_{\tilde{t}} \tilde{\phi}=\nu \partial_{\tilde{x}}^{2} \tilde{\phi}+\frac{\lambda}{2}\left(\partial_{\tilde{x}} \tilde{\phi}\right)^{2}+\sqrt{\mathcal{D}} \xi_{1},
$$

and it was originally proposed [46] to describe the growth of interfaces. Its scaling behavior at low energies (and assuming an infinite system and stationary regime) is characterized by the correlation function

$$
\Delta \tilde{\phi}_{\tilde{x}, \tilde{t}}^{2} \equiv\left\langle[\tilde{\phi}(\tilde{x}, \tilde{t})-\tilde{\phi}(0,0)]^{2}\right\rangle
$$

and by two exponents $\chi$ and $z$ which determine the asymptotic behavior of the spatial and temporal correlations, respectively, according to $\Delta \tilde{\phi}_{\tilde{x}, 0}^{2} \sim \tilde{x}^{2 \chi}$ and $\Delta \tilde{\phi}_{0, \tilde{t}}^{2} \sim \tilde{t}^{2 \chi / z}$.

In $1 \mathrm{D}$ we have $\chi=1 / 2$ for the roughness exponent and $z=3 / 2$ for the dynamical exponent; even more precisely, it holds that

$$
\Delta \tilde{\phi}_{\tilde{x}, \tilde{t}}^{2}=\left(\frac{1}{2} \lambda A^{2} t\right)^{2 / 3} g_{\mathrm{KPZ}}\left(\frac{\tilde{x}}{\left(2 \lambda^{2} A \tilde{t}^{2}\right)^{1 / 3}}\right),
$$

where $A=\mathcal{D} / 2 \nu$ and $\Delta \tilde{\phi}_{\tilde{x}, 0}^{2}=A|\tilde{x}|$ is the variance of $\partial_{\tilde{x}} \tilde{\phi}$. The universal function $g_{\mathrm{KPZ}}$ is known exactly [56], and we here recall its limiting values $g_{\mathrm{KPZ}}(u)-2|u| \rightarrow 0$ for $u \rightarrow$ $\infty$ and $g_{\mathrm{KPZ}}(u) \rightarrow 1.150 \ldots$ for $u \rightarrow 0$. As a consequence, the equal-time correlation function has the random walk 
form $\Delta \tilde{\phi}_{\tilde{x}, 0}^{2}=(A / 2)|\tilde{x}|$, which is insensitive to the $\mathrm{KPZ}$ nonlinearity $\lambda$ and can be captured by a linearized Bogoliubov analysis. In other words, looking at only the functional form of the spatial correlations, the dynamics is not distinguishable from the one of the linear $(\lambda=0)$ Edwards-Wilkinson (EW) model. In both cases, one has $\chi=1 / 2$, which corresponds to an exponential decay of the spatial coherence $[40,43]$. A difference is instead visible in the spatiotemporal correlations, which have different exponents in the two models, namely, $z=2$ in the linear EW model and $z=3 / 2$ in KPZ.

A numerical illustration of the collapse of the coherence functions to the scaling form (13) is given later on in Fig. 6(b) in comparison with the topological lasing case. Even though it goes beyond the present work, it is interesting to recall that the crossover from EW to KPZ physics is shown in Ref. [42] to be slower in the presence of a nonlinear refractive index.

\section{Linewidth of extended lasers}

The KPZ results reviewed in the previous subsection apply to spatially infinite systems in the long-time limit but are not of direct applicability to the concrete problem of the emission linewidth of a realistic laser device which necessarily has a finite spatial extension. To this purpose, the crucial quantity to study is the time dependence of the equal-space correlator

$$
g^{(1)}(t)=\frac{1}{n_{0}}\left|\left\langle\psi^{*}(x, t) \psi(x, 0)\right\rangle\right|,
$$

which characterizes the temporal coherence of the emission. The dependence of $g^{(1)}$ on $x$ is dropped, since we are considering a spatially uniform system.

\section{Linearized Bogoliubov prediction}

Within a Bogoliubov approximation where the theory is linearized around the mean-field steady state, modes of different momenta are decoupled. For a discrete lattice, one obtains with simple algebra that

$$
\begin{aligned}
\left\langle[\phi(x, t)-\phi(x, 0)]^{2}\right\rangle & =\frac{1}{N_{x}} \sum_{x}\left\langle[\phi(x, t)-\phi(x, 0)]^{2}\right\rangle \\
& =\frac{1}{n_{0} N_{x}} \sum_{k} \bar{D}_{k} \int_{0}^{t} d t^{\prime} e^{-2 i \omega_{k}\left(t-t^{\prime}\right)},
\end{aligned}
$$

where the effective drift coefficients $\bar{D}_{k}$ are determined by the shape of the Bogoliubov modes and tend to $D$ in the long-wavelength limit $k \rightarrow 0$. In this same limit, the lowest Bogoliubov mode has a diffusive character with $\omega_{k} \simeq$ $-i \gamma_{k}=-i J^{2} \Gamma^{-1} k^{4}$ [53]. The factor $1 / N_{x}$ in front of Eq. (15) can be interpreted by viewing the white spatial noise as randomly drawing noise realizations with a given $k$ and unit strength at each site. The probability to pick a given mode is then $1 / N_{x}$. Note that the "ultraslow decay of fluctuations" that is numerically observed in Ref. [30] is a consequence of the softness of the diffusive Goldstone branch and, thus, a general feature of spatially extended lasers.

In a spatially finite system where $k$ is quantized, only the $k=0$ mode gives a finite contribution at long times, proportional to $|t|$; the contribution of all other modes decays instead exponentially with time. From this result, one immediately obtains the expression of the BogoliubovSchawlow-Townes coherence time

$$
\tau_{c}=\tau_{\mathrm{ST}}=2 \gamma_{\mathrm{ST}}^{-1}=\frac{2 n_{0} N_{x}}{D}
$$

that generalizes the Schawlow-Townes phase diffusion to the case of a finite laser array, $n_{0} N_{x}$ being equal to the total number of photons.

The situation is a bit different for an infinite array. In this case, the sum over discrete $k$ modes has to be replaced by an integral $k$. This replacement yields the Bogoliubov prediction $g^{(1)}(t) \sim e^{-B|t|^{3 / 4}}$, where $B$ is a constant. The slower power-law decay stems from the fact that the specific $k=0$ mode is now occurring with probability zero.

For the sake of completeness, it is worth noting that a different scaling would be found in the presence of a nonlinear refractive index. In this case, the imaginary part of the Bogoliubov frequency would scale as $\gamma_{k} \propto k^{2}$ in the longwavelength $k \rightarrow 0$ limit [53], leading to a slower decay $g^{(1)}(t) \sim e^{-B|t|^{1 / 2}}$ in an infinite one-dimensional system.

\section{Nonlinear KPZ effects}

All the results discussed in the previous subsection are based on a linearized Bogoliubov approximation where different modes are decoupled. Of course, we know that this approximation is not adequate for an infinite or large enough system, where nonlinear KPZ features set in.

For an infinite system, a stretched exponential behavior

$$
g^{(1)}(t) \sim e^{-B|t|^{2 \beta}}
$$

is predicted in Ref. [43], with a universal $2 \beta=2 \chi / z=2 / 3$ and a nonuniversal value of the constant $B$.

If the system is sufficiently large but finite, the Bogoliubov approximation breaks down, but the spontaneously broken $\mathrm{U}(1)$ symmetry still imposes that the coherence must decay at long times at least as fast as a pure exponential, $g^{(1)}(t) \sim e^{-|t| / \tau_{c}}$. In this case, we expect that the KPZ physics typical of the infinite chain should remain visible only for intermediate times, up to a saturation time scaling as $\left(N_{x}\right)^{z}$.

These arguments on the functional form of the temporal decay of coherence are quantitatively illustrated in Fig. 1, where we display the temporal correlation function computed by numerically solving Eq. (7) for three different 

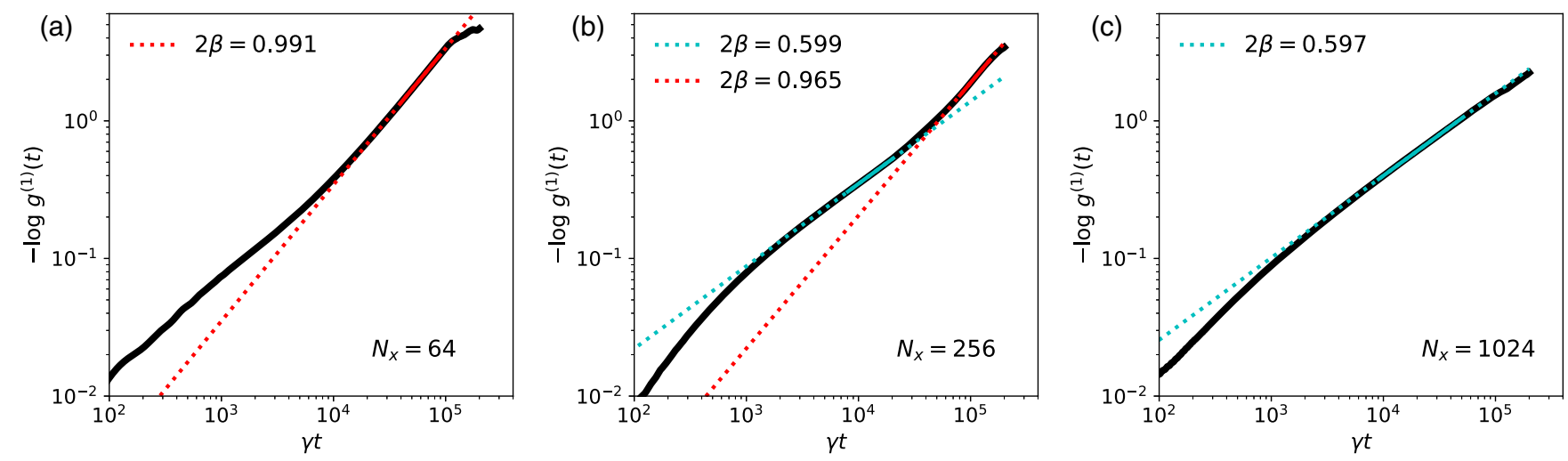

FIG. 1. Kardar-Parisi-Zhang to Schawlow-Townes crossover in the temporal coherence of finite, one-dimensional laser arrays. The numerical prediction of Eq. (7) for the logarithm of the equal-space time correlation function $-\log g^{(1)}(t)$ is plotted in $\log \log$ scale as a function of time for increasing system sizes $N_{x}=64$ (left), $N_{x}=256$ (middle), and $N_{x}=1024$ (right). In all cases, lasing occurs around $k_{x}^{\text {las }}=0$. For a given temporal window, the decay of the coherence is dominated by a Schawlow-Townes-like diffusion $g^{(1)}(t) \sim e^{-B|t|}$ for small sizes (a) and by the KPZ behavior $g^{(1)}(t) \sim e^{-B|t|^{2 / 3}}$ for large sizes (c). The crossover between the two regimes is visible for intermediate sizes (b). The cyan and red lines are fits of $\log \left[-\log g^{(1)}(t)\right]$ with functions of the form $2 \beta \log |t|+B^{\prime}$. Other system parameters: $J=0.5 \gamma, P=2 \gamma, n_{S}=1000$, and $D=\gamma$.

system sizes $N_{x}=64,256,1024:$ The thick black line shows $-\log g^{(1)}(t)$, while the red and cyan lines are linear fits in the $\log \log$ scale of the plot. Keeping the same observation window, for small system sizes the temporal decay of the coherence $g^{(1)}(t)$ is mainly diffusive and follows an exponential law [Fig. 1(a)]. For very large sizes [Fig. 1(c)], the exponential Schawlow-Townes behavior is pushed at very long times so that only the KPZ stretched exponential $g^{(1)}(t) \sim e^{-B|t|^{2 / 3}}$ is clearly visible in the time window displayed in the plot. An attempt to see the xponents of both regimes on a single plot is shown in the plot for an intermediate size shown in Fig. 1(b): While a hint of them is visible, a complete separation of the two regimes would require very large system sizes and very long observation times, which is numerically very demanding [57].

The KPZ scaling of $g^{(1)}(t)$ at intermediate times is a clear indication of the crucial role of nonlinear coupling between modes in determining the phase dynamics. While the linearized Bogoliubov theory predicts the (qualitatively correct) exponential form of the decay of coherence at long times, it is natural to wonder whether the KPZ nonlinear couplings are responsible for any quantitative deviation of the coherence time from the Bogoliubov-SchawlowTownes prediction (16).

This issue is numerically investigated [58] in Fig. 2, where we plot the numerical result for the coherence time $\tau_{c}$ in one-dimensional arrays of increasing sizes for two different values of the intersite coupling $J=5 \gamma$ (blue) and $J=0.5 \gamma$ (cyan). To better highlight the KPZ features, we normalize the coherence time to the single-site Schawlow-Townes coherence time $\tau_{\mathrm{ST}, 1} \equiv$ $\tau_{\mathrm{ST}} / N_{x}=\left(2 n_{0} / D\right)$. For all parameter choices, the coherence time follows the Bogoliubov scaling proportional to $N_{x}$ until a certain critical size numerically compatible with the scaling of $l^{*} \sim J^{4 / 7}$ given in Eq. (9), after which its increase with $N_{x}$ occurs at a much slower rate.

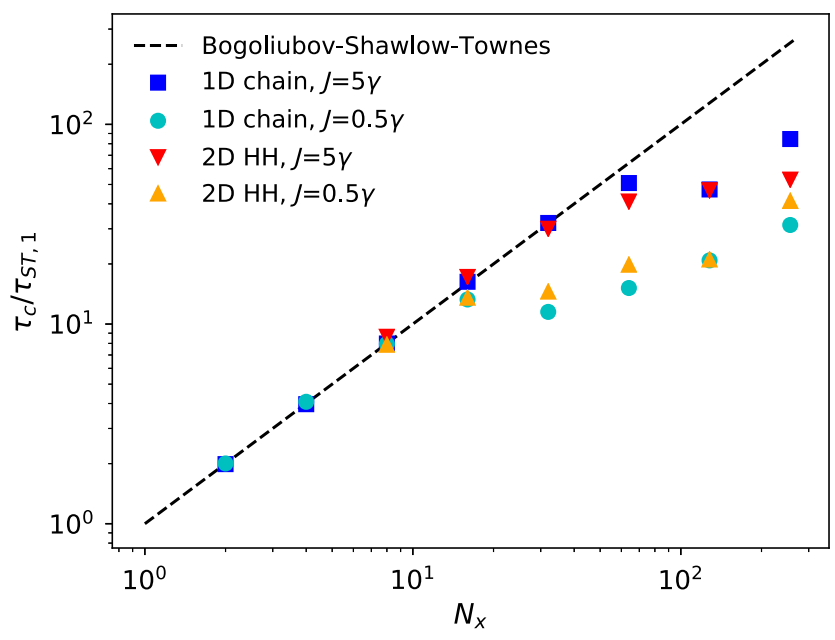

FIG. 2. Scaling of the coherence time with system size $N_{x}$. The coherence time $\tau_{c}$ is extracted from the long-time exponential decay of coherence for different systems. Blue and cyan markers refer to the one-dimensional, topologically trivial arrays of laser resonators of Eq. (7) with different values of the intersite coupling $J=5 \gamma$ and $J=0.5 \gamma$, respectively. The red and orange markers refer to the topological laser case of Eq. (20) for the same two values of $J$. For each point, the value of the coherence time $\tau_{c}$ is normalized to an effective single-site coherence time $\tau_{\mathrm{ST}, 1}$. For the 1D array, this value is defined by the Schawlow-Townes formula (6). For the topological 2D array, it is given by $\tau_{\mathrm{ST}, 1} \equiv \tau_{\mathrm{ST}} / N_{x}$, with $\tau_{\mathrm{ST}}$ being the Bogoliubov-Schawlow-Townes prediction (32) that includes the (small) Petermann correction. 
This marked deviation is indeed expected and can be understood looking at the KPZ equation (11): The total phase drift is the $\tilde{k}=0$ part of the phase field, which can be decomposed in two statistically independent contributions:

$$
\tilde{\phi}(\tilde{x}, \tilde{t})=\tilde{\phi}_{0}(\tilde{t})+\tilde{\phi}^{\prime}(\tilde{x}, \tilde{t}) \text {. }
$$

Here, $\tilde{\phi}_{0}$ accounts for the global phase evolution generated by the $\tilde{k}=0$ component of noise,

$$
\partial_{\tilde{t}} \tilde{\phi}_{0} \equiv \sqrt{\mathcal{D}} \xi_{1}(\tilde{k}=0, \tilde{t}),
$$

and yields the Schawlow-Townes drift. Even though the equation for $\tilde{\phi}^{\prime}(\tilde{x}, \tilde{t})$ is independent of $\xi_{1}(\tilde{k}=0, \tilde{t})$, an additional evolution of $\tilde{\phi}^{\prime}(\tilde{k}=0, \tilde{t})$ is induced by the finite $\tilde{k}$ components $\tilde{\phi}^{\prime}(\tilde{k} \neq 0, \tilde{t})$ of the phase field via the KPZ nonlinearity $\lambda$. The additional phase noise induced by this nonlinear coupling is responsible for the deviation of the coherence time from the Bogoliubov-Schawlow-Townes prediction visible in Fig. 2. The complex behavior of $\tau_{c}\left(N_{x}\right)$ shown in the figure suggests that a quantitative explanation of the phenomenon requires a nonperturbative analysis that is the subject of future work. In particular, the value of the exponent $\rho$ characterizing the large $N_{x}$ dependence $\tau_{c} \sim N_{x}^{\rho}$ is expected to be $\rho=1 / 2$ for the present 1D case [59].

Notice that in any finite system the coherence decays exponentially in time independently of the dimensionality of the lattice; even when spatial long-range order can be established, a linewidth enhancement is expected with $\rho=1$ [59]. For completeness, we mention that nonlinear mode couplings are predicted in Ref. [60] to play a crucial role in the phase diffusion of equilibrium condensates but have a different physical origin in Beliaev processes driven by thermal fluctuations.

\section{HARPER-HOFSTADTER TOPOLOGICAL LASER}

As shown in recent theoretical $[18,30]$ and experimental $[19,20]$ works, it is possible to make the edge mode of a photonic topological insulator to lase by introducing a gain material into the device, while preserving the topological properties such as the chirality of the edge mode propagation and its topological robustness in circumventing defects without suffering backscattering. This section is devoted to the study of the spatiotemporal coherence properties of such a device. Within the usual semiclassical approach, quantum and classical noise is described by including a white noise term to the equation of motion of the field. While the overall behavior turns out to be very similar to the nontopological case studied in the previous section, some interesting consequences of the topological nature of the mode are found and highlighted.

\section{A. The model}

With no loss of generality, we focus our attention on the topological bands of a two-dimensional Harper-Hofstadter model. This most celebrated topological model is widely used in studies of topological photonics. By labeling with $x=1, \ldots, N_{x}$ and $y=1, \ldots, N_{y}$ the sites of the twodimensional lattice, the equations of motion for the field read

$$
\begin{aligned}
i \partial_{t} \psi_{x, y}= & -J\left[\psi_{x, y+1}+\psi_{x, y-1}+e^{-2 \pi i \alpha y} \psi_{x-1, y}+e^{2 \pi i \alpha y} \psi_{x+1, y}\right] \\
& +\frac{i}{2}\left[\frac{P \delta_{y, 1}}{1+n_{x, y} / n_{S}}-\gamma\right] \psi_{x, y}+\sqrt{2 D_{x, y}} \xi_{x, y}
\end{aligned}
$$

where $\alpha$ is the synthetic magnetic field flux per plaquette in units of the magnetic flux quantum. As in many previous works, we focus on the $\alpha=1 / 4$ case. To simplify the geometry, we consider a cylindrical lattice with periodic boundary conditions along the $x$ direction, and we introduce the gain medium on all sites of the $y=1$ edge. Inspired by the Wigner approach [4], we take the diffusion coefficient to have the form $D_{x, y}=\left(1+\delta_{y, 1}\right) \gamma / 2$. The stronger noise on the edge sites reflects the presence of gain and the consequent spontaneous emission processes. We, however,
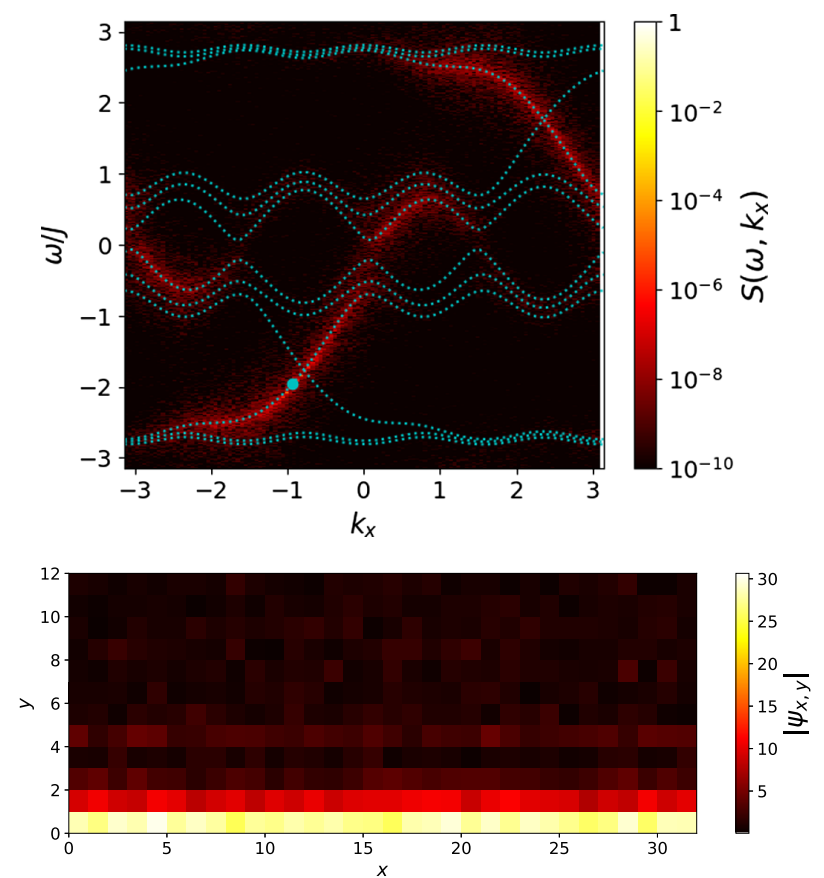

FIG. 3. Topological lasing. Lower: Typical snapshot of the field modulus distribution $|\psi(x, y)|$ at steady state. Upper: Wave-vector- and energy-resolved spectrum of the field on the $y=1$ edge. The dotted lines are the HarperHofstadter bands, and the spectral intensities are normalized to the laseremission at $\omega^{\text {las }} \simeq-9.774 \gamma, k_{x}^{\text {las }}=-2 \pi \frac{19}{128}$. Numerical calculations are performed according to Eq. (20) for a lattice of size $N_{x}=128, N_{y}=12$ with periodic boundary conditions along $x$ and a flux density $\alpha=1 / 4$. System parameters: $J=5 \gamma, P=2 \gamma, n_{s}=1000$, and $D_{x, y}=\left(1+\delta_{y, 1}\right) \gamma / 2$. 
check that our results remain qualitatively identical if different spatial distributions of $D_{x, y}$ are used. We also check that the statistical results that are discussed in the following are unchanged if different system geometries are considered, e.g., with open boundary conditions (see, e.g., Fig. S3 in Ref. [54]). We also mention the study [61] of the chiral fluctuations in a similar configuration but in the linear amplifier regime.

For the sake of completeness, let us first recall the properties of the underlying Harper-Hofstadter Hamiltonian in the absence of gain, losses, and noise. Within the Landau gauge used in Eq. (20), implementing periodic boundary conditions along the $x$ direction makes the system translationally invariant, so that it is convenient to label states by the wave vector $k_{x}$. The dispersion for our $\alpha=1 / 4$ case is shown by the cyan dotted lines in Fig. 3: It consists of four bands of bulk states delocalized over the whole system, plus two chiral modes localized on each edge and energetically located in the gaps within the bands. On the $y=1$ side per example, the edge state in the lower (upper) gap has positive (negative) group velocity, and vice versa for the other side. The fact that there are no two counterpropagating states on the same edge at the same frequency is at the origin of the topological protection.

We study the temporal evolution of the field according to the stochastic equations (20). Starting the simulation with zero field, noise triggers laser operation by spontaneously breaking the $\mathrm{U}(1)$ symmetry. A mean-field study of this physics in the absence of noise with random initial conditions is presented in Ref. [30]: At steady state, quasimonochromatic laser oscillation takes place in a mode which is randomly selected by the initial noise. The probability distribution is peaked at discrete frequencies roughly fixed by the eigenvalues of the underlying finite Harper-Hofstadter model. The distribution is symmetric with respect to zero frequency and has support in the energy gaps of the band structure. The maximum corresponds to the eigenstates that are most localized on the edge for which the effective gain is the strongest: As pointed out in Refs. [36,39], the $k_{x}$-dependent overlap of the HarperHofstadter eigenmode with the gain material determines a nontrivial dependence of the imaginary part of the dispersion.

The main features of the steady-state topological laser operation including noise are illustrated in Fig. 3. In the lower panel, we plot a typical example of the field modulus $\left|\psi_{x, y}\right|$ for a finite $N_{x}=32, N_{y}=12$ cylindrical lattice, showing localization of the mode on the edge. The upper panel reports instead the power spectral density $S\left(k_{x}, \omega\right)$ of the field $\psi_{x, 1}(t)$ on the $y=1$ edge: The narrow lasing mode is strongly saturated on this scale and is indicated by the cyan circle. Noise-induced fluctuations distribute themselves over all modes but are concentrated on the ones with largest overlap with the $y=1$ side, in particular, on the two edge states with opposite chiralities. While the spectral distribution roughly follows the dispersion of the optical modes in the underlying passive Harper-Hofstadter model indicated as a cyan dotted line, a complete theory requires a Bogoliubov analysis of the collective modes. This requirement is addressed in Ref. [62] and is the subject of the forthcoming work in Ref. [39].

\section{B. Chiral motion of the phase fluctuations}

To characterize the spatiotemporal coherence of the emission, we consider the fluctuations of the phase of the one-dimensional field living on the amplifying boundary of the Harper-Hofstadter lattice, $\psi(x, t) \equiv \psi_{x, 1}(t)$.

In the steady state of laser operation, the phase displays slow fluctuations around a carrier wave vector $k_{x}^{\text {las }}$ and frequency $\omega^{\text {las: }}$ The former can be extracted from the
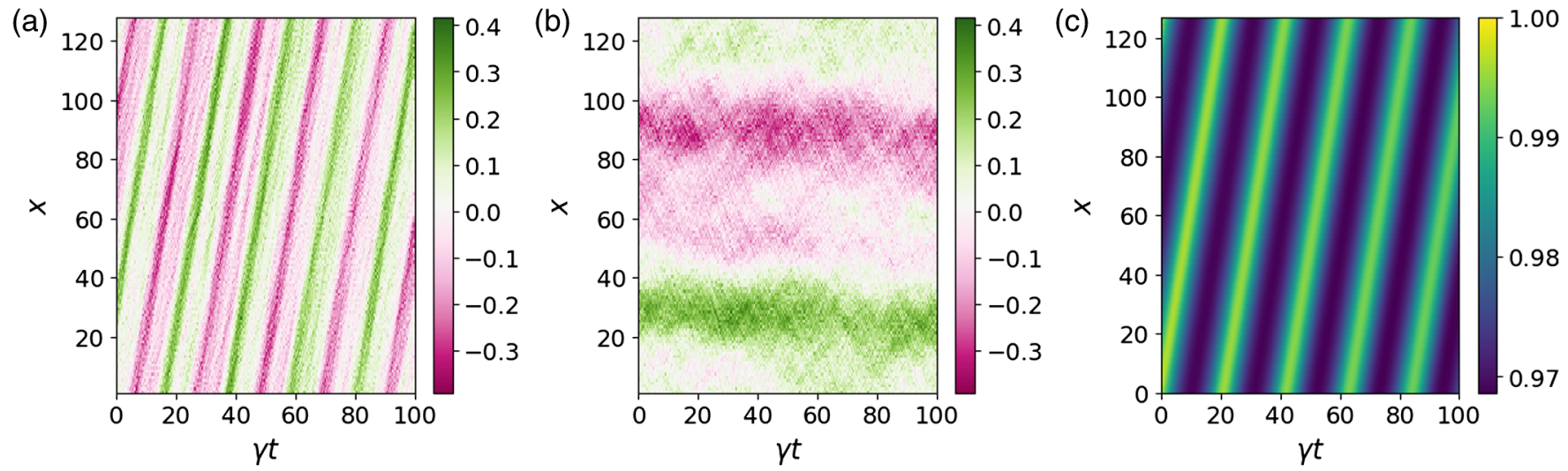

FIG. 4. Chiral motion of the phase fluctuations. (a) Spatiotemporal plot of the slowly varying phase of a random realization of $\psi_{\mathrm{sl}}(x, t)$, showing that the fluctuations chirally move around the system. (b) Spatiotemporal plot of the phase of $\psi_{\mathrm{CM}}(x, t)$ in the comoving frame: Now the fluctuations are observed in their natural frame of reference and evolve slowly. (c) Correlation function $g^{(1)}(x, t)$ : Since the fluctuations move at $v_{g}$, after a time $t$ the field in $x$ is correlated with the one in $x+v_{g} t$. System parameters: $N_{x}=128, N_{y}=12, J=5 \gamma, P=2 \gamma, n_{s}=1000$, and $D=2 \gamma$. 
(spatial) winding number of the phase around the system, and the latter can be determined by fitting the evolution of the field phase on single sites. In the spectrum of Fig. 3, they are indicated by the position of the cyan circle. While a precise determination of these quantities can be important from the applicative point of view, they are somehow uninteresting from the statistical mechanics point of view, since they are mostly determined by the deterministic dynamics of the device and are weakly affected by the fluctuations.

In order to remove the carrier frequency and wave vector and concentrate on the stochastic fluctuations, we define the slowly varying field

$$
\psi_{\mathrm{sl}}(x, t) \equiv e^{-i\left(k_{x}^{\mathrm{las}} x-\omega^{\mathrm{las}} t\right)} \psi(x, t) .
$$

Looking at the phase of a typical realization of $\psi_{\mathrm{sl}}(x, t)$ shown in Fig. 4(a), we easily recognize a phase fluctuation pattern that moves at a constant velocity and gets slowly distorted. The drift velocity can be inferred from the dispersion $\omega_{\mathrm{em}}\left(k_{x}\right)$ of the lasing chiral edge mode, which has group velocity $v_{g}=\left(d \omega_{\mathrm{em}} / d k_{x}\right)\left(k_{x}^{\text {las }}\right)$ and curvature $J_{\text {eff }}\left(k_{x}^{\text {las }}\right)=\frac{1}{2}\left(d^{2} \omega_{\text {em }} / d k_{x}^{2}\right)\left(k_{x}^{\text {las }}\right)<J$.

In order to focus on the intrinsic dynamics of the phase fluctuations, we plot in Fig. 4(b) a typical realization of the phase evolution seen from the moving frame at $v_{g}$ :

$$
\psi_{\mathrm{CM}}(x, t) \equiv \psi_{\mathrm{sl}}\left(x+v_{g} t, t\right) .
$$

For the relatively strong intersite coupling $J=5 \gamma$ and relatively small system size $N_{x}=128$, the phase fluctuations develop very slowly and remain quite small. Their magnitude gets larger if the mean intensity $n_{0}$ is reduced, the intersite coupling $J$ is reduced, or larger systems are considered. This situation is discussed in Sec. III D.

While the transformation to $\psi_{\mathrm{CM}}(x, t)$ allows for a direct visualization of the phase dynamics, it is also possible to study the fluctuations circulating along the edge by computing the space-time correlation function of the original field,

$$
g^{(1)}(x, t)=\frac{\left|\left\langle\psi^{*}(x, t) \psi(0,0)\right\rangle\right|}{\sqrt{\langle n(x)\rangle\langle n(0)\rangle}}
$$

where the average is taken over the noise and invariance under temporal $t$ and spatial $x$ translations is assumed. This analysis requires no preliminary estimate of $k_{x}^{\text {las }}$ and $\omega^{\text {las }}$ and is our workhorse in the next sections. As is apparent looking at the smooth stripes in Fig. 4(c), the analysis of $g^{(1)}(x, t)$ is the cleanest way to extract the velocity at which fluctuations travel. The result $v_{q} \simeq 6.07 \gamma a$ is perfectly compatible with the group velocity $v_{g} \simeq 6.08 \mathrm{\gamma a}$ obtained from the linear dispersion of the chiral edge mode (see also Fig. S4 in Ref. [54]).

\section{Correlated side peaks in the emission spectrum}

A complete understanding of the spectral distribution $\left|\psi\left(k_{x}, \omega\right)\right|^{2}$ obtained by spatiotemporal Fourier transform and shown in Fig. 3(b) requires inspecting the Bogoliubov modes on top of the noiseless lasing state. This inspection is the subject of a forthcoming work [39].

For the moment, we restrict our discussion to a few simple yet important remarks on the emission spectrum from each site. In Fig. 5(a), we show the emission spectrum defined as

$$
S(\omega)=\frac{1}{N_{x}} \sum_{x}|\psi(x, \omega)|^{2}
$$

for the parameters and lasing point shown in Fig. 3(b). In addition to the main lasing peak, the emission spectrum displays a comblike structure with a series of symmetric side peaks: The frequency spacing is determined by the quantization of the momentum along the periodic direction and is approximatively $v_{g}\left(2 \pi / N_{x}\right)$.

The visibility of the comb is not merely due to the existence of eigenstates at those specific values of the frequency, but their population by noise is enhanced by correlations. This enhancement is illustrated in Fig. 5(b), where we show the normalized momentum-space intensityintensity correlation function

$$
R^{(2)}\left(k_{x}, k_{x}^{\prime}\right)=\frac{\left\langle n_{k_{x}} n_{k_{x}^{\prime}}\right\rangle}{\sqrt{\left\langle n_{k_{x}}^{2}\right\rangle\left\langle n_{k_{x}^{\prime}}^{2}\right\rangle}} .
$$

Here, the momentum-space densities $n_{k_{x}}$ are evaluated at the same time over the whole edge: $n_{k_{x}}(t)=\left|\psi\left(k_{x}, t\right)\right|^{2}$.

Several features are visible in this plot. The diagonal line for $k_{x}=k_{x}^{\prime}$ is due to a trivial self-correlation, and $R^{(2)}$ is here equal to 1 . For generic pairs of modes, the thermal


FIG. 5. Correlated side peaks in the emission spectrum. (a) Spectrum of the field on a single resonator located on the amplified edge. The spacing of the side peaks is determined by the quantization of the wave vector $k_{x}$ around the periodic direction. The side peaks are generated by parametric scattering processes from the lasing mode into pairs of symmetrically located modes. (b) Color plot of the momentum-space correlation function (25) in the $k_{x}, k_{x}^{\prime}$ plane showing-among others-strong correlations along the antidiagonal, that is, within pairs of symmetrically located modes. 
$g^{(2)}\left(k_{x}\right)=2$ character of all $k_{x} \neq k_{x}^{\text {las }}$ modes implies that the background value is $R^{(2)}=0.5$. When one (two) modes coincide with the lasing one, $R^{(2)}$ is equal to $1 / \sqrt{2}(1)$, which explains the vertical and horizontal stripes and the central peak. The most interesting feature is the stripe on the antidiagonal, corresponding to correlations between symmetrically located modes such that $k_{x}+k_{x}^{\prime}=2 k_{x}^{\text {las }}$. For the first two pairs of side peaks, this correlation is nearly perfect, indicating that these modes are populated in pairs by parametric scattering processes.

Of course, such correlations are not specific of topological systems but can be observed also in the trivial systems studied in the previous Sec. II, albeit with a suppressed intensity due to the curvature of the dispersion. In analogy to exciton-polariton systems pumped around the magic angle [50], the magnitude of these parametric correlations is strongest if lasing is made to operate around the inflection point of the dispersion.

\section{KPZ spatiotemporal correlations}

The discussion of the emission spectrum presented in the previous subsection gives first hints of the complexity of the fluctuation dynamics in an extended device. Here, we build a complete theoretical picture of the spatiotemporal coherence of the topological laser.

Since the field is concentrated along the one-dimensional edges of the system and its transverse profile closely follows the one of the linear edge mode, it is natural to describe the dynamics in terms of a one-dimensional equation. Sitting in the comoving frame, this can be written in the form

$$
\begin{aligned}
i \partial_{t} \psi_{\mathrm{CM}}(x, t)= & \left(-J_{\mathrm{eff}}+i \eta\right) \partial_{x}^{2} \psi_{\mathrm{CM}} \\
& +\frac{i}{2}\left[\frac{P_{\mathrm{eff}}}{1+n_{S}\left|\psi_{\mathrm{CM}}\right|^{2}}-\gamma\right] \psi_{\mathrm{CM}}+\sqrt{2 D} \xi
\end{aligned}
$$

Here, $J_{\text {eff }}$ is given by the curvature of the bare HarperHofstadter topological mode, $P_{\text {eff }}$ is chosen to retrieve the numerical mean intensity $n_{0}$ on the edge, and $\eta$ accounts phenomenologically for the $k$-dependent localization of the lasing mode on the edge of the lattice and the consequent $k$ dependence of gain. A rigorous account of this dimensional reduction procedure is presented in Ref. [39]. We mention that a finite $\eta$ would arise also in the 1D chain if a dissipative coupling described by a nonvanishing imaginary part of $J[34,63,64]$ were considered.

Assuming a fast relaxation of the intensity fluctuations, we can then restrict our attention to the dynamics of the phase. By neglecting terms containing four derivatives (both the linear, Galilean-preserving ones and the nonlinear, Galilean-breaking ones as shown in Supplemental Material [54]), one gets to a motion equation for the phase of the KPZ form:

$$
\partial_{t} \phi=\eta \partial_{x}^{2} \phi-\frac{J_{\text {eff }}^{2}}{\Gamma} \partial_{x}^{4} \phi-J_{\text {eff }}\left(\partial_{x} \phi\right)^{2}+\sqrt{\frac{D}{n_{0}}} \xi .
$$

Since $\eta$ is the less controlled parameter of the model, we do not perform the usual KPZ rescaling [43] to yield an equation containing the effective nonlinearity as the only parameter. Rather, we rely on the rescaling Eqs. (9) with the effective parameters $J \rightarrow J_{\text {eff }}, P \rightarrow P_{\text {eff }}$; this transformation does not depend on $\eta$ [65] and yields

$$
\partial_{\tilde{t}} \tilde{\phi}=\frac{\eta t_{*}}{l_{*}^{2}} \partial_{\tilde{x}}^{2} \tilde{\phi}-\partial_{\tilde{x}}^{4} \tilde{\phi}+\left(\partial_{\tilde{x}} \tilde{\phi}\right)^{2}+\tilde{\xi} \text {. }
$$

In these units, we then expect the KPZ nonlinearity to be close to $\lambda=2$; this value is also protected from renormalization induced by the quartic derivative term in the KSE [55]. It is thus natural to expect that the coherence of the topological laser will closely resemble the one of the generic extended laser discussed in Sec. II. In what follows, we proceed to numerically verify this statement on simulations of the stochastic laser equations in the topological two-dimensional lattice.

Based on our previous discussion, we expect that the KPZ universal dynamics occurs, in a lattice of $N_{x}$ sites, on timescales shorter than the saturation time $\sim N_{x}^{z}$ (after which the Schawlow-Townes-like behavior described above sets in) but larger than the timescales where the linear Bogoliubov dynamics and nonuniversal effects dominate. Having a sizable window to observe KPZ physics then requires the system to be large enough; precisely, it should be at least $N_{x} a \gg \sqrt{2} \pi l^{*}$ [40]. We thus consider a large system of length $N_{x}=1024$ with periodic boundary conditions along $x$ and with $N_{y}=12$ points along the open direction $y$. In order to clearly observe KPZ physics while keeping intensity fluctuations within $15 \%$ and having a tractable system size, it is beneficial to use a small intersite coupling $J=0.5 \gamma$.

One may argue that such a value of the coupling $J$ (and, thus, of the topological gap) is comparable with the bare linewidth $\gamma$. Such narrow topological gaps are very relevant for experimental implementations [20], but it is not a priori obvious whether in this regime the chiral edge modes survive losses. While this issue is indeed serious to observe chiral edge propagation in passive systems, it is a crucial result of laser theory that the laser linewidth above threshold can be orders of magnitude smaller than $\gamma$. Our numerical simulations confirm stable lasing into the chiral edge mode even for small $J / \gamma$; in particular, the robustness of topological lasing is verified in the presence of a strong defect on the edge, as reported in Fig. S5 [54].

To numerically highlight the KPZ physics, we perform 20 simulations of the full two-dimensional lattice of duration $\gamma T=5 \times 10^{5}$, starting from a plane wave with the wave vector value for which the Harper-Hofstadter eigenstate is most localized on $y=1$, that is, 
$k_{x}=-2 \pi \frac{155}{1024}$. For each run, after a suitable equilibration time, the correlation function $\left\langle\psi^{*}(x, t+\bar{t}) \psi(0, \bar{t})\right\rangle$ on the edge site is computed and then averaged over the 20 trials to yield $g^{(1)}(x, t)$. The typical dynamics occurring in a time $\gamma T=2 \times 10^{4}$ is depicted in Fig. 6(a), where the phase of the field $\psi_{\mathrm{CM}}(x, t)$ along the edge is shown in the comoving frame: A structure similar to the fractal structure of interface growth can be recognized.

Defining the correlation function in the comoving frame as

$$
g_{\mathrm{CM}}^{(1)}(x, t)=\frac{\left|\left\langle\psi_{\mathrm{CM}}^{*}(x, t) \psi_{\mathrm{CM}}(0,0)\right\rangle\right|}{\sqrt{\left\langle n_{\mathrm{CM}}(x)\right\rangle\left\langle n_{\mathrm{CM}}(0)\right\rangle}}
$$

and the rescaled correlator as

$$
\tilde{C}\left(\tilde{t}, \tilde{x}^{z}\right) \equiv-2\left(\phi^{*}\right)^{-2} \tilde{x}^{-2 \chi} \log g_{\mathrm{CM}}^{(1)}(\tilde{x}, \tilde{t}),
$$

KPZ universality requires that

$\tilde{C}(\tilde{x}, \tilde{t})=\tilde{C}\left(\tilde{t} / \tilde{x}^{z}\right)=\left(\frac{1}{2} \lambda A^{2} s\right)^{2 / 3} g_{\mathrm{KPZ}}\left(\frac{1}{\left(2 \lambda^{2} A s^{2}\right)^{1 / 3}}\right)$

with $s=\tilde{t} / \tilde{x}^{z}$ and $z=3 / 2$ on the right-hand side and $g_{\mathrm{KPZ}}$ a universal function discussed in Sec. II B. In particular, one has $\tilde{C}(0)=A$.

A series of curves of $\tilde{C}(\tilde{x}, \tilde{t})$ for $x= \pm 30, \ldots, \pm 160$ is plotted as orange lines in Fig. 6(b) as a function of $\tilde{t} / \tilde{x}^{z}$ [to correct for the finite size effects, we actually plot $\tilde{C}(\tilde{x}, \tilde{t}) /\left(1-|x| / N_{x}\right)$, as illustrated in Fig. S6 [54] ]. The collapse of these lines onto a single curve demonstrates that within an excellent approximation $\tilde{C}$ depends only on $\tilde{t} / \tilde{x}^{z}$, as expected from the KPZ scaling. This result for the topological laser is to be compared to an analogous analysis for a trivial one-dimensional array with the same physical parameters lasing in the $k=0$ mode as discussed in Sec. II B. Also in this case, the different lines (cyan) collapse on a single curve, confirming the expected KPZ scaling.

The crucial point about Fig. 6(b) is that the phase, the space, and the time are rescaled by the $\phi^{*}, l^{*}$, and $t^{*}$ values, respectively, obtained by using the effective masses and gain parameters: For the topological laser, $J_{\text {eff }}$ is the curvature of the Harper-Hofstadter band at the lasing point, and $P_{\text {eff }}$ is chosen in order to reproduce the observed intensity on the edge. For instance, $J_{\text {eff }} \simeq 0.319 \gamma$ and, hence, $l^{*} \simeq 1.92$ here.

As already mentioned, the renormalization group analysis [55] predicts for the nontopological 1D array lasing in the $k^{\text {las }}=0$ mode that the rescaled KSE Eq. (11) flows to the low-energy effective KPZ theory Eq. (10) with $\lambda=2$, since, thanks to the Galilean invariance holding for $\mathrm{KSE}$ and KPZ equations, the nonlinear coupling is not
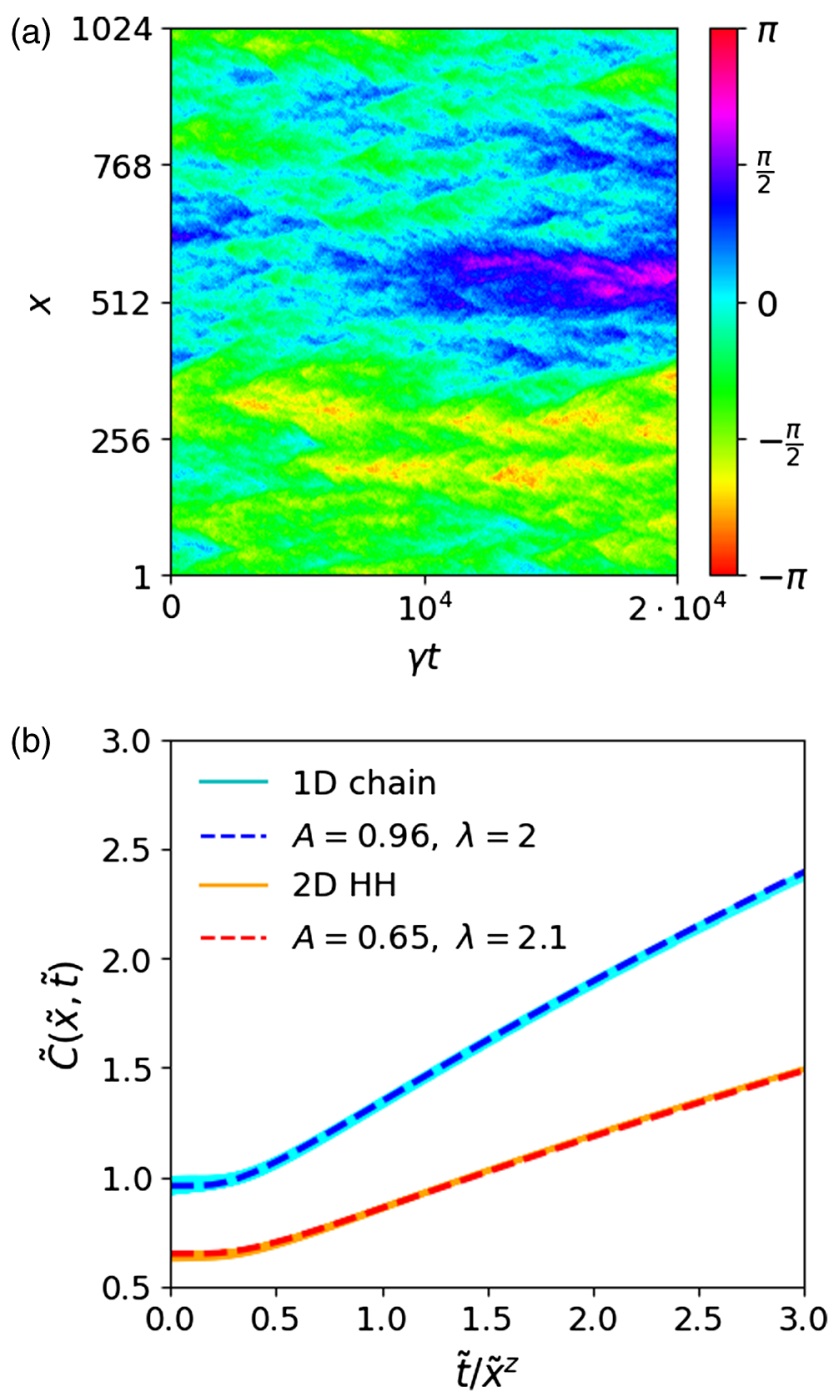

FIG. 6. KPZ dynamics of topological lasing. (a) Typical example of the steady-state space-time dynamics of the phase of the field $\psi_{\mathrm{CM}}(x, t)$ on the system edge seen from the comoving frame. (b) Correlators $\tilde{C}\left(\tilde{t}, \tilde{x}^{z}\right)$ for $x= \pm 30, \ldots, \pm 160$ (small lines) for the topological device lasing into the $k_{x}=-2 \pi \frac{155}{1024}$ mode (orange) and for the nontopological one-dimensional array lasing in the $k_{x}=0$ mode (cyan). Red and blue dashed lines indicate the KPZ universal function (31) on which all curves collapse. System parameters: $N_{x}=1024, J=0.5 \gamma, P=2 \gamma$, $n_{S}=1000$, and $D_{x, y}=\gamma / 2\left(1+\delta_{y, 1}\right)$.

renormalized. This result is confirmed by our simulations, which show that the rescaled correlation functions indeed collapse to a unique curve as shown in Fig. 6(b), and this curve is excellently fitted (blue dashed line) using Eq. (31) with $\lambda=2$ and $A=0.96$, as expected from the Galilean invariance argument.

For the topological laser, the curves again collapse onto a single curve, which is well fitted using $\lambda=2.1$ and $A=0.65$ (red dashed line). Note that an upper bound for the fitted value of the nonlinearity is provided by the value $\lambda=2.3$ with $A=0.63$. 
In contrast to the nontopological case, for the topological laser there is no a priori guarantee that a Galilean invariant KSE holds microscopically; to the contrary, an analysis along the lines of Refs. $[40,41]$ suggests that a rescaling with $J_{\text {eff }}$ still yields a microscopic $\lambda=2$, but other terms should also be added in Eq. (10), e.g., of the kind $\partial_{x}^{2} \phi\left(\partial_{x} \phi\right)^{2},\left(\partial_{x}^{2} \phi\right)^{2}, \partial_{x}^{3} \phi \partial_{x} \phi$, etc. These additional terms come from effective imaginary derivatives due to the $k_{x}$ dependent localization of the Harper-Hofstadter eigenstates on the edge; in particular, they break Galilean invariance, and one may expect that they significantly renormalize the effective KPZ parameters, since they contain the same number (four) of derivatives as the KSE term. However, it turns out from our numerics that the renormalization of $\lambda$ remains small.

Still, it is interesting to note that the curves for the topological laser (in the properly rescaled units) sit below the ones of the trivial one-dimensional laser array and are correspondingly fitted by a KPZ form with a lower $A$. This feature can be traced back to the imaginary term proportional to $\eta$ in Eq. (26) that accounts for the $k$ dependence of the edge mode penetration in the bulk. This term stabilizes the emission and makes the topological device more coherent than the 1D laser with the corresponding $J_{\text {eff }}$ and $P_{\text {eff }}$. The crucial role of $\eta$ in topological devices is apparent already at the level of Bogoliubov analysis [39], at least for class-A lasers. It is also remarkable that the very noisy field in the bulk that is visible, e.g., Fig. S2 of Ref. [54], does not impact the coherence of the edge mode.

We conclude this section with a brief remark on the experimental protocol to assess KPZ physics. The analysis of the correlation functions $g_{\mathrm{CM}}^{(1)}(x, t)$ shown in Fig. 6(b) is carried out in the reference frame comoving with the chiral mode. Typical experiments measure correlation functions between different times $g^{(1)}(x, t)$ and different points in the laboratory frame. However, since the correlation functions in the comoving and laboratory frames are simply related by $g_{\mathrm{CM}}^{(1)}(x, t)=g^{(1)}\left(x-v_{g} t, t\right)$, the interesting $g_{\mathrm{CM}}^{(1)}$ can be extracted by a straightforward postprocessing of $g^{(1)}(x, t)$ measured in the laboratory frame. Graphically, this processing amounts to tilting the correlation function in Fig. 4(c) with the suitable $v_{g}$ to have the maximum of $g^{(1)}(x, t)$ at $x=0$ for all times $t$ (see Fig. S4 [54]).

\section{E. Temporal coherence and linewidth}

After having confirmed that the long-time, large-distance spatiotemporal coherence of the topological laser is well described by the KPZ model, we now proceed to investigate the problem of the coherence time of a realistic, finite-size device. Rather than analyzing with high resolution the linewidth of the main spectral peak in Fig. 5(a), we work in real time, and, with a concrete optical experiment in mind, we monitor the temporal coherence of the emission from a given site. As compared to our discussion of the trivial case in Sec. II B, there is the complication that phase fluctuations undergo a chiral motion around the system, so the coherence function of a given site displays the strong temporal oscillations that are visible in Fig. 4(c).

\section{KPZ to Schawlow-Townes crossover}

As a first step, we illustrate the crossover between KPZ coherence decay and Schawlow-Townes for different system sizes $N_{x}$. In Fig. 7, we show the temporal evolution of the phase diffusion $-\log g^{(1)}(t)$ (thick solid black lines) and $-\log g_{\mathrm{CM}}^{(1)}(t) \equiv-\log g_{\mathrm{CM}}^{(1)}(0, t) \quad$ (thin dashed black lines) in a given temporal window for increasing $N_{x}=64,256,1024$. As expected, these curves show sharp
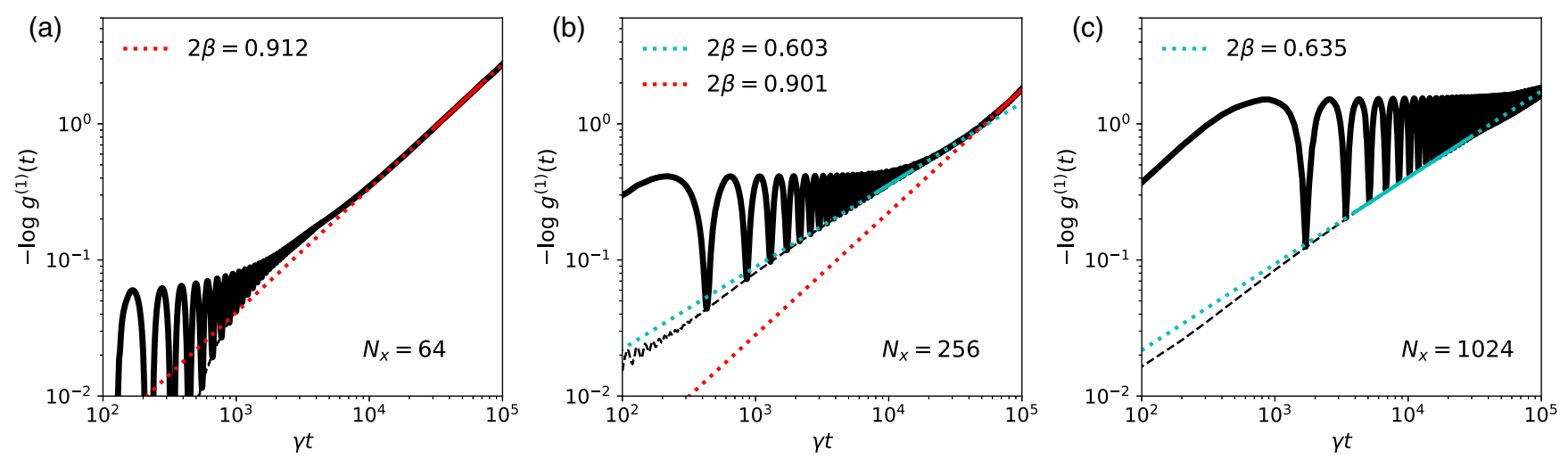

FIG. 7. Kardar-Parisi-Zhang to Schawlow-Townes crossover in a finite-size topological laser. This plot is the analog of Fig. 1 for the topological laser. The thick black lines correspond to the logarithm of the temporal correlation for a given site on the edge of the lattice. The thin dashed lines show the temporal evolution of the coherence $-\log g_{\mathrm{CM}}^{(1)}(t)$ seen from the reference frame comoving with the edge state. For increasing system sizes and a given temporal window, a crossover between an exponential (red fits) and a KPZ (cyan fits) decay of the coherence is observed. The amplitude of the oscillations is inversely proportional to the spatial coherence of the device. In the long-time limit, only the global phase matters, and the oscillations fade away. 
local minima of $-\log g^{(1)}(t)$ corresponding to local maxima of the coherence. As they originate from chiral motion of fluctuations around the system, these oscillations have a period $N_{x} / v_{g}$. The value of the coherence at these minima provides a discrete sampling of the equal-space coherence function $g_{\mathrm{CM}}^{(1)}(t)$. Similarly, the local maxima (minima of coherence) provide a sampling of $g_{\mathrm{CM}}^{(1)}\left(N_{x} / 2, t\right)$.

Looking at the envelope of the minima for the largest system, we see that the agreement of Fig. 7(c) with the KPZ result is very good. In particular, it makes a clear distinction from the predictions of the linear Edwards-Wilkinson model for which the exponent would be different. Note also that the oscillations in $-\log g^{(1)}(t)$ are well visible at short times but fade away at long times, where only the global phase of the field over the whole lattice matters and no oscillation is any longer visible. For the same reason, monitoring a resonator in the bulk would show a fast initial decay, but at long times the phase diffusion would recover the same value as on the edge, as sketched in Fig. S2 [54].

For the smaller systems, the different functional form shown in Fig. 7(a) displays an exponential decay of the late-time coherence. As one can see comparing with Fig. 7(b), the late-time exponential decay is always there; it is just pushed to extremely long times in the largest systems. This result is in close analogy with what we find in Sec. II C for the trivial system.

\section{Linearized Bogoliubov prediction for the linewidth}

The natural question is now to estimate the rate of this exponential decay and see how it scales with the size of the system. As a first step, we adopt a linearized Bogoliubov approach. For generic systems of $N$ sites (labeled as $\vec{x}$ ) of arbitrary dimensionality, let us call $\mathcal{L}_{\text {las }}$ the $2 N \times 2 N$ Bogoliubov matrix of the linearized dynamics on top of the lasing steady state. Let $V=\left\{V_{\vec{x} \sigma, p}\right\}$ be the invertible matrix which diagonalizes $\mathcal{L}_{\text {las }}$, where the pseudospin $\sigma=$ $\uparrow, \downarrow$ indicates the particle and hole components of the Bogoliubov problem and $p$ labels the $2 N$ eigenmodes. The Goldstone mode $V_{\vec{x} \sigma, G}$, that we assume to be unique with all other excitations having a finite lifetime, is the eigenstate with zero eigenvalue. As usual, its spatial shape follows the one of the lasing mode. The effective noise acting on the lasing mode is determined by the projection of the bare noise on it. For generality, we consider a position-dependent bare noise $D_{\vec{x}}$. Then, in the Bogoliubov approximation, the phase drift associated with the Goldstone mode is given by

$$
\left\langle\Delta_{t} \phi^{2}\right\rangle=\Phi_{G}^{2} \sum_{\vec{x}} D_{\vec{x}}\left(V^{-1}\right)_{G, \vec{x} \uparrow}\left(V^{-1}\right)_{G, \vec{x} \downarrow}|t|,
$$

where the summation represents the projection of the noise on the Goldstone mode and
$\Phi_{G}=\Phi_{G}(\vec{x})=\frac{-i}{n_{0}(\vec{x})}\left[\psi_{0}^{*}(\vec{x}) V_{\vec{x} \uparrow, G}-\psi_{0}(\vec{x}) V_{\vec{x} \downarrow, G}\right]$

is actually independent of $\vec{x}$ and provides the normalization of the Goldstone mode phase component.

For a system which is translationally invariant along $x$, the Bogoliubov matrix can be made block diagonal $\mathcal{L}_{\text {las }}=\bigoplus_{k_{x}} \mathcal{L}_{\text {las }}\left(k_{x}\right)$ with the transverse part of the Goldstone $V_{y \sigma, G}$ diagonalizing the $k_{x}=0$ block. Then, in Eq. (32), one should replace $\sum_{\vec{x}} \rightarrow N_{x} \sum_{y}, \psi_{0}(\vec{x})=$ $\psi_{0}(y) e^{i k_{x}^{\text {las } x}} \rightarrow \psi_{0}(y)$ and, choosing the standard normalization of the modes, $V_{\vec{x} \sigma, G} \rightarrow V_{y \sigma, G} / \sqrt{N_{x}}, \quad V_{G, \vec{x} \sigma}^{-1} \rightarrow$ $V_{G, y \sigma}^{-1} / \sqrt{N_{x}}$. This approach leads us to write $\tau_{\mathrm{ST}}=$ $N_{x} \tau_{\mathrm{ST}, 1}$, where $\tau_{\mathrm{ST}, 1}$ does not depend on $N_{x}$ but in a subtle and weak way via the discretization of $k_{x}^{\text {las }}$.

If $V V^{\dagger}=1$, a very clear expression holds for the Schawlow-Townes line:

$$
\gamma_{\mathrm{ST}}=\frac{\sum_{\vec{x}} D_{\vec{x}} n_{\vec{x}}}{n_{\mathrm{tot}}^{2}}=\frac{D_{n}}{n_{\mathrm{tot}}}
$$

with $n_{\text {tot }}=\sum_{\vec{x}} n_{\vec{x}} \quad$ and $D_{n}=\sum_{\vec{x}} D_{\vec{x}} n_{\vec{x}} / \sum_{\vec{x}} n_{\vec{x}}$. This expression is, for instance, the case of a spatially uniform, topologically trivial system, for which the different wave vectors decouple and the sector of $\mathcal{L}_{\text {las }}\left(k_{x}=0\right)$, corresponding to the lasing wave vector $\vec{k}_{\text {las }}$, is diagonalized by a $2 \times 2$ unitary matrix $V V^{\dagger}=1$, since modulus and phase are decoupled for the lasing mode.

The situation is a bit more complicated in the topological case: The $k_{x}=0$ sector has dimension $2 N_{y} \times 2 N_{y}$ and $V$ is not unitary $V V^{\dagger} \neq 1$, so the theory discussed above does not hold. However, for the considered parameters it turns out that $V V^{\dagger} \simeq 1$, so the approximate expression

$$
\tau_{\mathrm{ST}}=\frac{2}{\gamma_{\mathrm{ST}}} \simeq 2 \frac{\left[\sum_{x, y} n_{x, y}\right]^{2}}{\sum_{x, y} n_{x, y} D_{x, y}}=2 N_{x} \frac{\sum_{y} n_{1, y}}{D_{n}}
$$

is expected to provide an accurate approximation. In the standard laser theory, the nonorthogonality of $V$ is also known under the terminology of Petermann factor and excess noise $[47,48]$. The transverse Petermann factor, defined here as the ratio $\mathcal{K}=\left[\gamma_{\mathrm{ST}} /\left(D_{n} / n_{\text {tot }}\right)\right]$, for the topological laser device is around $\mathcal{K} \simeq 1.002$ for $J=5 \gamma$ and $\mathcal{K} \simeq 1.1$ for $J=0.5 \gamma$ (the difference to be attributed to gain guiding), meaning that, within the linearized Bogoliubov approximation, the laser emission is for all practical purposes determined only by the total number of photons in the device, which is the textbook, optimal case. This result confirms that topological guiding in the edge mode is immune from the linewidth-broadening effects typical of lasing in open resonators with gain guiding. 


\section{Numerical results for the linewidth}

In order to assess the validity range of the Bogoliubov calculation, numerical simulations of the stochastic equations are performed for the full two-dimensional model. The numerical predictions for the coherence time of the topological laser are shown by the red and orange triangles in Fig. 2 as a function of the system size. The dashed line shows the theoretical prediction Eq. (32). From these results, one concludes that the topological laser behaves again similarly to the topologically trivial one-dimensional laser array: On one hand, the agreement with the Bogoliubov-Schawlow-Townes model of phase diffusion is excellent for small $N_{x}$ and the coherence grows proportionally to $N_{x}$. On the other hand, a much slower growth of the coherence time with $N_{x}$ is found for larger systems.

As a final point, it is interesting to note that the Bogoliubov-Schawlow-Townes prediction that well captures the emission linewidth for small systems does not depend on $J_{\text {eff }}$ nor on the dispersion of the Bogoliubov modes at $k_{x} \neq 0$. On the other hand, the deviation observed for larger systems does strongly depend on $J_{\text {eff }}$, which pinpoints the crucial role of the KPZ nonlinearities illustrated above [66].

\section{LASING WITH ON-SITE DISORDER}

The general message of the previous section is that the coherence properties of a topological laser follow the same $\mathrm{KPZ}$ dynamics as the ones of a topologically trivial, onedimensional laser array. This conclusion is not restricted to the well-known KPZ features in the infinite system limit but also applies to the dependence of the coherence time on the system size and to its marked deviations from the Bogoliubov-Schawlow-Townes prediction.

In this section, we investigate the effect of static disorder on the coherence of the laser emission. A certain degree of fabrication imperfections and inhomogeneities is, in fact, expected to be always present in any real device. As we are now going to see, our numerical study points out a dramatically different behavior of topologically trivial vs topological systems: Disorder has a strong impact on the coherence of a topologically trivial system, a small amount of disorder being able to give a wide range of realization-dependent, chaotic, and multimode phenomena. On the other hand, the temporal coherence of a topological laser is robust against a sizable disorder, and emission remains well monochromatic as long as the disorder magnitude is not so large to close the topological gap.

\section{A. Lasing in a nontopological one-dimensional array with disorder}

We start by considering the effect of on-site disorder on the lasing properties of a nontopological array of resonators. We do not aim here to a general discussion of the theory of lasing in disordered systems or to make a connection with random lasers [67], but our purpose is just to provide a benchmark to assess the features of a topological laser.

Along all this section, a disordered potential is added to Eqs. (7) and (20) in the form

$$
i \partial_{t} \psi_{\vec{x}}=\cdots+W \mathcal{G}(0,1) \psi_{\vec{x}}
$$

where $\mathcal{G}(0,1)$ is a Gaussian random variable with mean 0 and variance 1 . For the sake of definiteness, we restrict here to the $N_{x}=128$ and $J=5 \gamma$ case. The lasing dynamics in the presence of disorder is, in general, very complex, but, since our ultimate goal is a qualitative comparison with the topological laser, we focus here on the coherence time of the system for various values of disorder $W$ and, in particular, on whether there is a clear threshold value of disorder above which coherence collapses.

For linear waves, the sensitivity of the eigenstates at a given energy to a static perturbation is proportional to the spectral density of states. Then, in order to have a fair comparison of the trivial and topological cases, we consider lasing both at $k^{\text {las }}=0$ and at $k^{\text {las }}=2 \pi \frac{13}{128}$. This latter case has a finite group velocity (and, hence, a density of states) comparable to the one of the chiral edge mode of the Harper-Hofstadter model in its central part, and for these

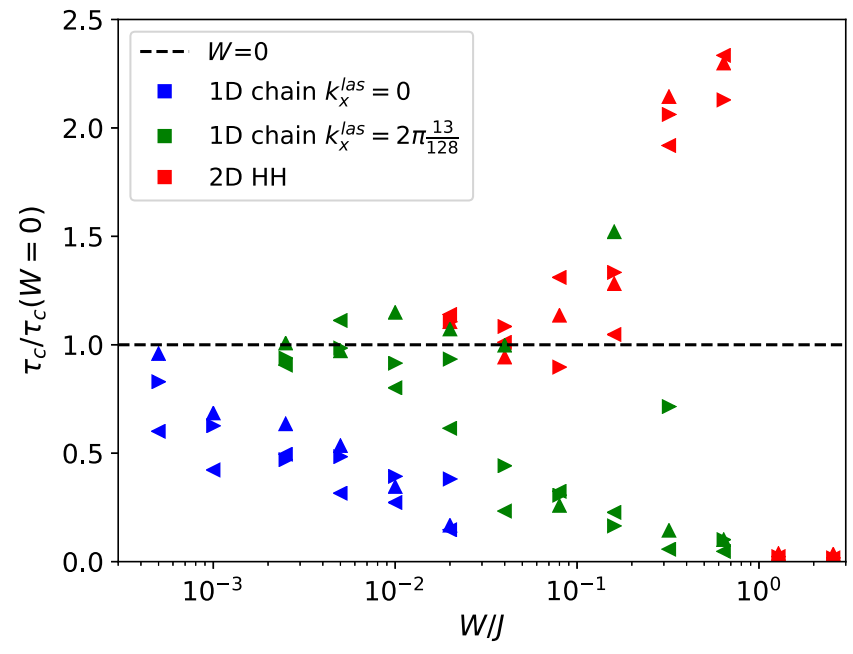

FIG. 8. Topological robustness of the temporal coherence. Plot of the coherence time normalized to the clean system value, as a function of the strength $W$ of the disorder. Different markers refer to different realizations of disorder. Blue and green markers are for a nontopological one-dimensional laser array lasing at $k^{\text {las }}=0$ (blue) or $k^{\text {las }}=2 \pi \frac{13}{128}$ (green). Red markers are for the topological laser with periodic boundary conditions (squares and triangles) and with open boundary conditions (crosses). The same marker shapes correspond to the same spatial distribution of the disorder potential except for the overall strength $W$. 
reasons we propose it as the proper benchmark for the topological laser.

In Fig. 8, we plot the coherence time normalized to the value in the absence of disorder. In particular, we use an exponential fit to extract the coherence time $\tau_{c}$ for each site [an exponential fit is used even if the shape of $g^{(1)}(t)$ is, in general, very complex], and we plot the average over the lattice. Markers with the same shape indicate that the same realization of disorder and the same initial conditions are used, while only the overall strength factor $W$ is varied.

Looking at the blue and green points in the plot, we see that already a very small disorder has a marked impact on the coherence time of the device. As expected, the threshold value depends on $k_{x}^{\text {las }}$, as visible comparing the blue dataset for lasing at $k^{\text {las }}=0$ and the green dataset for a finite $k^{\text {las }}$ where the density of states is smaller. While a detailed description of the variety of possible behaviors for different disorder realizations goes beyond the scope of this work, some illustrative examples are shown in Supplemental Material [54]. In particular, for intermediate disorder $W \sim 0.08 \mathrm{~J}$, laser operation may get fragmented with different portions of the sample lasing at different frequencies [see Figs. S7(c) and S7(d) in Ref. [54] ]. Calculations for different realizations of disorder suggest that coherence can be greatly reduced even in the absence of fragmentation [as shown in Fig. S7(b)] and without affecting the intensity of the field, thus showing that there is no general one-byone correspondence between the power slope efficiency and the robustness of coherence robustness to static disorder. Finally, curious nonmonotonic behaviors can also be observed in some other realizations.

\section{B. Lasing in a topological disordered system}

The same protocol is repeated for the topological laser on a $N_{x}=128$ times $N_{y}=12$ stripe with $J=5 \gamma$, periodic boundary conditions along $y$ and gain localized on the $y=1$ row of sites. The results are reported as red datasets in Fig. 8. Simulations are also performed with fully open boundary conditions and gain distributed along the whole edge, yielding the same conclusions. In contrast to the nontopological case discussed in the previous subsection, the behavior of the topological laser remains quite regular in the presence of disorder, and different realizations show very similar features.

For weak disorder strengths $W$, disorder has a negligible impact: As naively expected, scattering on defects is topologically suppressed, as the laser field in the chiral edge mode is able to continuously travel around the system almost undisturbed. The temporal and spatial coherence properties of the clean system are, thus, very well preserved (as apparent in Fig. S8 [54]). For strong disorders, topological protection breaks down, and the coherence displays a marked threshold at a value of disorder $W_{c} \sim J$, that is, on the order of the topological gap of the underlying
Harper-Hofstadter model. Beyond this value, spatial and temporal coherence are rapidly lost.

The most intriguing regime is for intermediate values of the disorder strength, where we observe a surprising and systematic enhancement of the temporal coherence. A tentative explanation for this expected behavior can be put forward in terms of the KPZ dynamics. Even though they are too small to destroy the topology, these values of $W / J$ are strong enough to hamper the nonlinear phase dynamics that is responsible for the deviation from the Bogoliubov-Schawlow-Townes prediction seen in Fig. 2. As a result, for intermediate $W$ the coherence time recovers the Bogoliubov-Schawlow-Townes prediction (32).

This interpretation is substantiated by the analysis reported in Fig. 9. As a first step, in Fig. 9(a), we plot the real and imaginary parts of the lowest Bogoliubov eigenfrequencies $[39,62]$ for a few realizations of disorder and different disorder strengths indicated by the shape and color of the markers. As usual in such calculations, the computation consists in reaching the noiseless steady state via the deterministic evolution in the presence of disorder and then finding the eigenmodes of the linearized evolution around the steady state by diagonalizing Bogoliubov matrix. From the plot, it is apparent how disorder has a strong effect in reducing the lifetime of the excitation modes. Physically, this behavior can be understood in terms of the fluctuation modes being pushed by the disorder deeper into the nonamplifying bulk of the lattice. Since the Bogoliubov-Schawlow-Townes coherence time is not sensitive to the decay rate of fluctuation modes, this effect is, however, not enough to explain the numerical observation shown in Fig. 8: As reported in the inset, a computation using the linearized Bogoliubov-Schawlow-Townes recipe of Eq. (35) rather predicts a slightly decreased coherence time for increasing disorder.

On the other hand, as the precursor of KPZ physics in a linearized analysis is typically given by the softening of the Bogoliubov modes, the faster decaying modes reported in Fig. 9(a) hint at a suppression of the effective nonlinear coupling responsible for the KPZ-broadened linewidth. This intuition is quantitatively tested by repeating our calculation of the coherence time for different system sizes. As we have seen in Fig. 2, the KPZ nonlinearity has, in fact, no effect on small systems, but it dramatically reduces the coherence of large systems.

Figures 9(b) and 9(c) fully confirm this mechanism. For the small $N_{x}=32$ system size for which Fig. 2 shows an accurate agreement with Schawlow-Townes, we find in Fig. 9(b) that no enhancement is present and the coherence time remains nearly constant up to the threshold value $W_{c}$ for which topological protection is broken. Figure $9(\mathrm{c})$ shows the same data on a different scale, with the coherence time normalized to the Bogoliubov-Schawlow-Townes prediction plotted in the inset in Fig. 9(a). For small lattices $N_{x}=32$, the relative coherence time $\tau_{c} / \tau_{\mathrm{ST}} \approx 1$ up to $W_{c}$, 

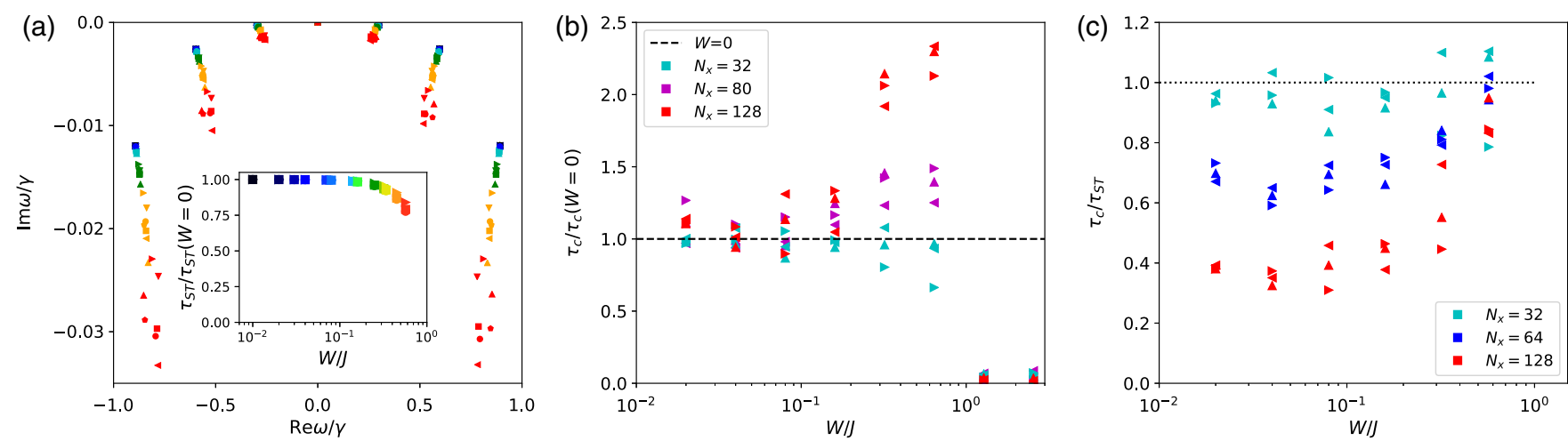

FIG. 9. (a) Bogoliubov modes $\omega_{\lambda}$ in the Goldstone dome for a $N_{x}=128, J=5 \gamma$ topological array in the presence of disorder, plotted as $\operatorname{Im} \omega_{\lambda}$ versus $\operatorname{Re} \omega_{\lambda}$. Different marker shapes correspond to different realizations of disorder, and colder to warmer colors point at increasing values of $W / J$. Remarkably, the lifetime of the Bogoliubov modes is significantly reduced for $W \sim 0.5 \mathrm{~J}$, so that longer times and larger arrays are required to observe appreciable nonlinear effects in the phase dynamics. Inset: Bogoliubov-Schawlow-Townes prediction for the coherence time $\tau_{\mathrm{ST}}$ for increasing disorder strength $W / J$, plotted in units of the coherence time in the clean sample. (b) Plot of the ratio $\tau_{c}(W) / \tau_{c}(0)$ as a function of the disorder strength $W / J$ for different system sizes $N_{x}=32,80,128$; in particular, note how the enhanced coherence time at intermediate $W / J$ is observed only for large enough systems. (c) Plot of the ratio $\tau_{c}(W) / \tau_{\mathrm{ST}}(W)$ as a function of disorder strength $W / J$ for different system sizes $N_{x}=32,64,128$, showing how the linewidth recovers the Bogoliubov prediction for smaller arrays and for stronger disorder close to the threshold.

showing that the Bogoliubov-Schawlow-Townes prediction is accurate and the KPZ nonlinearity plays no role. For the large system with $N_{x}=128$, the relative coherence time $\tau_{c} / \tau_{\mathrm{ST}}$ is suppressed at small disorders by the KPZ nonlinearity and recovers the unit value only as the threshold $W_{c}$ is approached and the KPZ nonlinearity is no longer effective. We check that these considerations remain valid for different noise distribution $D_{x, y}$, as displayed in Fig. S9.

As a last point, it is worth commenting on the dependence of these results on the specific value of $k^{\text {las }}$, that is, the winding number $k^{\text {las }} N_{x} /(2 \pi)$ of the lasing mode. For a nontopological one-dimensional system, we see in the blue and green datasets of Fig. 8 that lasing at different $k^{\text {las }}$ gives very different robustness to disorder. On the other hand, we find that topological lasing is quite insensitive to the specific value of $k^{\text {las }}$ that can be chosen for laser operation [30]. The simulations of the topological laser can thus be started with arbitrary initial conditions on the field, finding almost identical behaviors at the steady state. This result further demonstrates that the topological device is able to automatically reach a stable and coherent steady-state emission.

\section{DISCUSSION}

As stated in the introduction, our calculations are based on a simple model that brings together two main ingredients: laser oscillation, meant as an archetype of nonlinear physics, and topologically protected chiral edge states. Within this idealized model, we give a rather complete treatment of the spatiotemporal coherence properties of topological laser emission. While our predictions may directly apply to class-A laser devices, many additional elements are neglected that would be essential to describe those semiconductor laser that are of strongest interest for applications. In this subsection, we discuss to what extent our results are expected to apply to realistic devices, in particular, the recent semiconductor-based realizations $[17,19,20,22,23,27]$, and in which properties the main deviations are expected to occur.

A first crucial issue to be carefully considered is the actual validity of the tight-binding approximation for a specific topological photonic system and the possibility of a complex nonlinear dependence of the tight-binding parameters on the circulating light intensity. The tight-binding approximation performed in our calculations consists in solving the nonlinear dynamics of the field on a discrete lattice of coupled single-mode resonators instead of dealing $a b$ initio with the full nonlinear Maxwell equations in the microscopic geometry of the underlying topological structure. To this latter purpose, powerful methods have been developed in the past decades, e.g., the so-called SALTsteady-state $a b$ initio laser theory [38]— but they remain computationally very expensive compared to the tightbinding model: They allow one to compute the deterministic steady state and then compute the Schawlow-Townes linewidth within the linearized approach but have never been pushed to more subtle spatiotemporal features in spatially extended systems. This computation is a computationally very challenging task that will be subject of further investigations.

Concerning the gain mechanism, in this work we focus our attention on an idealized gain medium where the optical nonlinearity reduces to gain saturation and does not affect the real part of the refractive index. Furthermore, we also assume that the gain medium has a temporally 
instantaneous response. This approximation is good for a gain medium with a fast relaxation rate, in which the electronic dynamics in the medium can be adiabatically eliminated in favor of the electromagnetic field dynamics. While this approximation is appropriate for so-called classA lasers, making quantitative predictions for specific semiconductor laser devices requires extending our theory to go beyond these approximations, which will be the subject of future work.

Still, in spite of all these complexities of real-life systems, we do not expect that the additional elements will introduce major changes to the qualitative features of the long-time, large-distance physics. For instance, it is shown in Refs. $[40,43,45]$ that the characteristic length and timescales over which the KPZ regime is observed are, of course, modified in the presence of reactive optical nonlinearities, but not the universal scaling behavior.

But one must not forget that all our theory applies only as long as the additional features-in particular, the slow carrier dynamics - do not introduce dynamical instabilities akin to those predicted in Refs. $[36,39,68]$ or multimode lasing behaviors. Developing a theoretical framework able to predict the single- or many-mode behavior of a specific laser device and to understand the actual advantage of topology [69] in stabilizing single-mode emission is an extremely challenging task which will most likely require a full microscopic theory. In any case, inspection of the experimental spectra reported in Refs. $[18,20]$ agree with our numerical calculation in giving a stable single-mode emission, so this challenge does not appear to be a concern for existing experiments.

More specifically, it is well known that the details of the gain medium $[35,71]$ can have a sizable impact on the linewidth, but they typically do not change the exponential functional form of the long-time coherence decay. In particular, the intensity dependence of the refractive index is typically responsible of an additional linewidth-broadening effect as intensity fluctuations translate (often in a temporally nonlocal way determined by the carrier dynamics) into fluctuations of the cavity mode frequency and, thus, in a diffusion of the phase. The ensuing linewidthbroadening effect is quantified by the so-called Henry factor, which in semiconductor lasers can be as large as a few tens. In realistic topological lasers, we expect that this broadening source will sum up with the Petermann factor and the broadening factor arising from the nonlinear KPZ phase dynamics illustrated in Fig. 2.

The effect of a noninstantaneous response of the gain medium on the linewidth requires some distinctions. On one hand, a slow carrier dynamics breaking our adiabaticity assumption should not introduce any additional linewidthbroadening effect [35]. On the other hand, the restricted frequency band of gain that is often associated to a noninstantaneous response of the gain medium may enhance both spatial and temporal coherence, since lasing typically occurs at the point of maximum gain and the weaker amplification of fluctuating side modes results in a faster effective damping $\nu$ in Eq. (11) and $\eta$ in Eq. (27). A theoretical study of the role of a frequency-dependent amplification in favoring laser operation in the topological edge modes over bulk modes as observed in Ref. [20] will be the topic of a forthcoming publication [72].

Finally, even though in this work we have chosen the strength of the noise having in mind the ultimate limit set by spontaneous emission, our semiclassical analysis extends straightforwardly to include any additional source of noise which gives homogeneous broadening. In this way, one can deal with a wide range of practical applications where a Lorentzian linewidth is observed, possibly much broader than the Schawlow-Townes limit. In contrast, genuinely quantum fluctuation effects are expected to become important in the case of multimode lasing [73] and in the presence of strong optical nonlinearities. These interesting issues require a specific study; in particular, the role of topology in these regimes remains unexplored.

In summary, in this concluding section, we have discussed a number of physical effects that were neglected in our model but will need be taken into account to get a quantitative description of experiments. Still, since our conclusions on the spatiotemporal coherence are based on universal properties, we can anyway expect that they are independent of the underlying microscopic model, so they should extend to generic realizations of the topological laser concept by adjusting the effective length and timescales appearing in the correlation functions, irrespectively of the specific material platform under investigation. The only crucial assumption-apparently well verified in the recent experiments $[18,20]$ - is that no dynamical instabilities occur, so the dynamics can be described by a classical stochastic field oscillating at a unique carrier frequency, or in the laser terminology, that the emission is a single-mode one.

\section{CONCLUSIONS}

In this work, we have investigated the spatiotemporal coherence properties of arrays of coupled laser resonators, discussing analogies and differences between lasing in a nontopological one-dimensional chain and chiral edgestate lasing in a 2D topological Harper-Hofstadter lattice. A main focus of our work has been to clarify how the spatial fluctuations of a nonequilibrium classical field impact on its temporal coherence.

As a common feature of both nontopological and topological laser arrays, we have highlighted the crossover for growing observation times from a Kardar-Parisi-Zhang scaling of the temporal coherence to a Schawlow-Towneslike phase-diffusion regime. Furthermore, for growing system sizes the long-time phase diffusion rate displays a crossover from the standard Bogoliubov-SchawlowTownes linewidth with an optimal, near unity Petermann 
factor, to a faster decoherence determined by the nonlinear dynamics of spatial fluctuations encoded in the KPZ dynamics.

Crucial consequences of topology have instead been identified in disordered systems, where the chiral motion of the lasing mode entails a much larger resilience to fabrication imperfections. For the nontopological arrays, disorder is, in fact, able to spatially localize the lasing mode and/or break it into several disconnected and incoherent pieces. On the other hand, the topological protected chiral motion of the edge state of a topological laser device is able to phase lock the different sites and, thus, maintain the spatial and temporal coherence across the whole sample up to much larger values of the disorder strength on the order of the topological gap.

These results confirm the strong promise that topological lasers hold for practical optoelectronic applications where one needs to make an array of many lasers to phase lock and emit as a single laser. Also, on the fundamental science side, topological laser are extremely promising to suppress undesired spatial inhomogeneities and boundary effects in experimental studies of the critical properties of different nonequilibrium statistical models [74].

\section{ACKNOWLEDGMENTS}

We are grateful to Jacqueline Bloch, Leonie Canet, Alessio Chiocchetta, Aurelian Loirette-Pelous, Matteo Seclì, Moti Segev, and Davide Squizzato for useful discussions. The addition of Fig. 9 and of Sec. V were stimulated by the constructive feedback that we received from the referees, to whom we are strongly indebted. We acknowledge financial support from the H2020FETFLAG-2018-2020 project "PhoQuS" (No. 820392) and from the Provincia Autonoma di Trento. All numerical calculations were performed using the Julia programming language [75].

[1] A. E. Siegman, Lasers (Oxford University Press, New York, 1987).

[2] O. Svelto, Principles of Lasers (Springer, New York, 2010).

[3] H. Haken, Synergetics (Springer, Berlin, 1983).

[4] C. Gardiner and P. Zoller, Quantum Noise: A Handbook of Markovian and Non-Markovian Quantum Stochastic Methods with Applications to Quantum Optics (Springer Science, New York, 2004), Vol. 56.

[5] A. Chiocchetta, A. Gambassi, and I. Carusotto, Laser Operation and Bose-Einstein Condensation: Analogies and Differences, in Universal Themes of Bose-Einstein Condensation, edited by N. P. Proukakis, D. W. Snoke, and P.B. Littlewood (Cambridge University Press, Cambridge, England, 2017), pp. 409-423.

[6] J. Keeling, L. M. Sieberer, E. Altman, L. Chen, S. Diehl, and J. Toner, Superfluidity and Phase Correlations of Driven Dissipative Condensates, in Universal Themes of
Bose-Einstein Condensation, edited by N.P. Proukakis, D. W. Snoke, and P. B. Littlewood (Cambridge University Press, Cambridge, England, 2017), pp. 205-230.

[7] L. Lu, J. D. Joannopoulos, and M. Soljačić, Topological Photonics, Nat. Photonics 8, 821 (2014).

[8] T. Ozawa, H. M. Price, A. Amo, N. Goldman, M. Hafezi, L. Lu, M. C. Rechtsman, D. Schuster, J. Simon, O. Zilberberg, and I. Carusotto, Topological Photonics, Rev. Mod. Phys. 91, 015006 (2019).

[9] F. D. M. Haldane and S. Raghu, Possible Realization of Directional Optical Waveguides in Photonic Crystals with Broken Time-Reversal Symmetry, Phys. Rev. Lett. 100, 013904 (2008).

[10] Z. Wang, Y. Chong, J. D. Joannopoulos, and M. Soljačić, Observation of Unidirectional Backscattering-Immune Topological Electromagnetic States, Nature (London) 461, 772 (2009).

[11] M. C. Rechtsman, J. M. Zeuner, Y. Plotnik, Y. Lumer, D. Podolsky, F. Dreisow, S. Nolte, M. Segev, and A. Szameit, Photonic Floquet Topological Insulators, Nature (London) 496, 196 (2013).

[12] M. Hafezi, S. Mittal, J. Fan, A. Migdall, and J. M. Taylor, Imaging Topological Edge States in Silicon Photonics, Nat. Photonics 7, 1001 (2013).

[13] Y. Lumer, Y. Plotnik, M. C. Rechtsman, and M. Segev, SelfLocalized States in Photonic Topological Insulators, Phys. Rev. Lett. 111, 243905 (2013).

[14] S. Mukherjee and M. C. Rechtsman, Observation of Floquet Solitons in a Topological Bandgap, Science 368, 856 (2020).

[15] D. Smirnova, D. Leykam, Y. Chong, and Y. Kivshar, Nonlinear Topological Photonics, Appl. Phys. Rev. 7, 021306 (2020).

[16] G. Harari, M. A. Bandres, Y. Lumer, Y. Plotnik, D. N. Christodoulides, and M. Segev, Topological Lasers, in Proceedings of the Conference on Lasers and ElectroOptics (Optical Society of America, Washington, D.C., 2016), p. FM3A.3, https://www.osapublishing.org/abstract .cfm?uri=CLEO_QELS-2016-FM3A.3.

[17] S. Wittek, G. Harari, M. A. Bandres, H. Hodaei, M. Parto, P. Aleahmad, M. Rechtsman, Y. D. Chong, D. N. Christodoulides, M. Khajavikhan, and M. Segev, Towards the Experimental Realization of the Topological Insulator Laser, in Proceedings of the Conference on Lasers and Electro-Optics (Optical Society of America, Washington, D.C., 2017), p. FTh1D.3.

[18] G. Harari, M. A. Bandres, Y. Lumer, M. C. Rechtsman, Y. D. Chong, M. Khajavikhan, D. N. Christodoulides, and M. Segev, Topological Insulator Laser: Theory, Science 359, eaar4003 (2018).

[19] M. A. Bandres, S. Wittek, G. Harari, M. Parto, J. Ren, M. Segev, D. N. Christodoulides, and M. Khajavikhan, Topological Insulator Laser: Experiments, Science 359, eaar4005 (2018).

[20] B. Bahari, A. Ndao, F. Vallini, A. El Amili, Y. Fainman, and B. Kanté, Nonreciprocal Lasing in Topological Cavities of Arbitrary Geometries, Science 358, 636 (2017).

[21] L. Pilozzi and C. Conti, Topological Lasing in Resonant Photonic Structures, Phys. Rev. B 93, 195317 (2016). 
[22] P. St-Jean, V. Goblot, E. Galopin, A. Lemaître, T. Ozawa, L. L. Gratiet, I. Sagnes, J. Bloch, and A. Amo, Lasing in Topological Edge States of a One-Dimensional Lattice, Nat. Photonics 11, 651 (2017).

[23] M. Parto, S. Wittek, H. Hodaei, G. Harari, M. A. Bandres, J. Ren, M. C. Rechtsman, M. Segev, D. N. Christodoulides, and M. Khajavikhan, Edge-Mode Lasing in 1D Topological Active Arrays, Phys. Rev. Lett. 120, 113901 (2018).

[24] C. Han, M. Lee, S. Callard, C. Seassal, and H. Jeon, Lasing at Topological Edge States in a Photonic Crystal 13 Nanocavity Dimer Array, Light Sci. Appl. 8, 40 (2019).

[25] Y. Ota, R. Katsumi, K. Watanabe, S. Iwamoto, and Y. Arakawa, Topological Photonic Crystal Nanocavity Laser, Commun. Phys. 1, 86 (2018).

[26] Y. Ota, K. Takata, T. Ozawa, A. Amo, Z. Jia, B. Kante, M. Notomi, Y. Arakawa, and S. Iwamoto, Active Topological Photonics, Nanophotonics 9, 547 (2020).

[27] Y. Zeng, U. Chattopadhyay, B. Zhu, B. Qiang, J. Li, Y. Jin, L. Li, A. G. Davies, E. H. Linfield, B. Zhang, Y. Chong, and Q. J. Wang, Electrically Pumped Topological Laser with Valley Edge Modes, Nature (London) 578, 246 (2020).

[28] Z.-K. Shao, H.-Z. Chen, S. Wang, X.-R. Mao, Z.-Q. Yang, S.-L. Wang, X.-X. Wang, X. Hu, and R.-M. Ma, A HighPerformance Topological Bulk Laser Based on BandInversion-Induced Reflection, Nat. Nanotechnol. 15, 67 (2020).

[29] Z. Yang, E. Lustig, G. Harari, Y. Plotnik, Y. Lumer, M. A. Bandres, and M. Segev, Mode-Locked Topological Insulator Laser Utilizing Synthetic Dimensions, Phys. Rev. X 10, 011059 (2020).

[30] M. Seclì, M. Capone, and I. Carusotto, Theory of Chiral Edge State Lasing in a Two-Dimensional Topological System, Phys. Rev. Research 1, 033148 (2019).

[31] A. L. Schawlow and C. H. Townes, Infrared and Optical Masers, Phys. Rev. 112, 1940 (1958).

[32] E. Kapon, J. Katz, and A. Yariv, Supermode Analysis of Phase-Locked Arrays of Semiconductor Lasers, Opt. Lett. 9 , 125 (1984).

[33] J. K. Butler, D. E. Ackley, and D. Botez, Coupled-Mode Analysis of Phase-Locked Injection Laser Arrays, Appl. Phys. Lett. 44, 293 (1984).

[34] S. Longhi and L. Feng, Invited Article: Mitigation of Dynamical Instabilities in Laser Arrays via Non-Hermitian Coupling, APL Photonics 3, 060802 (2018).

[35] A. Pick, A. Cerjan, D. Liu, A. W. Rodriguez, A. D. Stone, Y. D. Chong, and S. G. Johnson, Ab Initio Multimode Linewidth Theory for Arbitrary Inhomogeneous Laser Cavities, Phys. Rev. A 91, 063806 (2015).

[36] S. Longhi, Y. Kominis, and V. Kovanis, Presence of Temporal Dynamical Instabilities in Topological Insulator Lasers, Europhys. Lett. 122, 14004 (2018).

[37] C. Henry, Theory of the Linewidth of Semiconductor Lasers, IEEE J. Quantum Electron. 18, 259 (1982).

[38] H. E. Türeci, A. D. Stone, and B. Collier, Self-Consistent Multimode Lasing Theory for Complex or Random Lasing Media, Phys. Rev. A 74, 043822 (2006).

[39] A. Loirette-Pelous, Theory of Chiral Edge State Lasing in a Two-Dimensional Topological System, arXiv:1912.03911.
[40] V. N. Gladilin, K. Ji, and M. Wouters, Spatial Coherence of Weakly Interacting One-Dimensional Nonequilibrium Bosonic Quantum Fluids, Phys. Rev. A 90, 023615 (2014).

[41] E. Altman, L. M. Sieberer, L. Chen, S. Diehl, and J. Toner, Two-Dimensional Superfluidity of Exciton Polaritons Requires Strong Anisotropy, Phys. Rev. X 5, 011017 (2015).

[42] K. Ji, V. N. Gladilin, and M. Wouters, Temporal Coherence of One-Dimensional Nonequilibrium Quantum Fluids, Phys. Rev. B 91, 045301 (2015).

[43] L. He, L. M. Sieberer, E. Altman, and S. Diehl, Scaling Properties of One-Dimensional Driven-Dissipative Condensates, Phys. Rev. B 92, 155307 (2015).

[44] R. Lauter, A. Mitra, and F. Marquardt, From Kardar-ParisiZhang Scaling to Explosive Desynchronization in Arrays of Limit-Cycle Oscillators, Phys. Rev. E 96, 012220 (2017).

[45] D. Squizzato, L. Canet, and A. Minguzzi, Kardar-ParisiZhang Universality in the Phase Distributions of OneDimensional Exciton-Polaritons, Phys. Rev. B 97, 195453 (2018).

[46] M. Kardar, G. Parisi, and Y.-C. Zhang, Dynamic Scaling of Growing Interfaces, Phys. Rev. Lett. 56, 889 (1986).

[47] A. E. Siegman, Excess Spontaneous Emission in NonHermitian Optical Systems. I. Laser Amplifiers, Phys. Rev. A 39, 1253 (1989).

[48] A. E. Siegman, Excess Spontaneous Emission in NonHermitian Optical Systems. II. Laser Oscillators, Phys. Rev. A 39, 1264 (1989).

[49] J. Kasprzak, M. Richard, S. Kundermann, A. Baas, P. Jeambrun, J. Keeling, F. Marchetti, M. Szymańska, R. André, J. Staehli et al., Bose-Einstein Condensation of Exciton Polaritons, Nature (London) 443, 409 (2006).

[50] I. Carusotto and C. Ciuti, Quantum Fluids of Light, Rev. Mod. Phys. 85, 299 (2013).

[51] M. Lax, Classical Noise. V. Noise in Self-Sustained Oscillators, Phys. Rev. 160, 290 (1967).

[52] M. Scully and M. Zubairy, Quantum Optics (Cambridge University Press, Cambridge, England, 1997).

[53] M. Wouters and I. Carusotto, Excitations in a Nonequilibrium Bose-Einstein Condensate of Exciton Polaritons, Phys. Rev. Lett. 99, 140402 (2007).

[54] See Supplemental Material at http://link.aps.org/ supplemental/10.1103/PhysRevX.10.041060 for details on the derivation of the modulus-phase equations, numerical determination of the linewidth, open boundary conditions, finite size effects and examples of disordered lasing.

[55] K. Ueno, H. Sakaguchi, and M. Okamura, RenormalizationGroup and Numerical Analysis of a Noisy KuramotoSivashinsky Equation in $1+1$ Dimensions, Phys. Rev. E 71, 046138 (2005).

[56] M. Prähofer and H. Spohn, Exact Scaling Functions for One-Dimensional Stationary KPZ Growth, J. Stat. Phys. 115, 255 (2004).

[57] Note that the small downward deviations that are visible on the black curves next to the right edge of the plotting window [in particular, in Fig. 1(a)] are a numerical artifact due to the large statistical spread of the late-time points. An explanation of its origin is given in Sec. SII of Supplemental Material [54].

[58] Note that the numerical effort required for these calculations grows up rapidly with $N_{x}$, since one has to access the 
dynamics at very long times, larger than the KPZ saturation time scaling as $\sim N_{x}^{3 / 2}$. For this reason, a statistical analysis of the errors is restricted to the $N_{x}=128, J=5 \gamma$ case, as expanded in Sec. SII of Supplemental Material [54].

[59] I. Amelio, A. Chiocchetta, and I. Carusotto, Theory of the Linewidth of $1 D$ Non-Equilibrium Quasi-Condensates (to be published).

[60] A. Sinatra, Y. Castin, and E. Witkowska, Coherence Time of a Bose-Einstein Condensate, Phys. Rev. A 80, 033614 (2009).

[61] V. Peano, M. Houde, F. Marquardt, and A. A. Clerk, Topological Quantum Fluctuations and Traveling Wave Amplifiers, Phys. Rev. X 6, 041026 (2016).

[62] P. Zapletal, B. Galilo, and A. Nunnenkamp, Long-Lived Elementary Excitations and Light Coherence in Topological Lasers, Optica 7, 1045 (2020).

[63] I. L. Aleiner, B. L. Altshuler, and Y. G. Rubo, Radiative Coupling and Weak Lasing of Exciton-Polariton Condensates, Phys. Rev. B 85, 121301(R) (2012).

[64] S. L. Harrison, H. Sigurdsson, and P. G. Lagoudakis, Synchronization in Optically Trapped Polariton StuartLandau Networks, Phys. Rev. B 101, 155402 (2020).

[65] Actually, also the dependence on $\Gamma_{\text {eff }}$ is such that, given the measured $n_{0}$, any uncertainty on the value of $P_{\text {eff }}$ affects only the KPZ coefficient of the Laplacian.

[66] For this plot, we choose the $k_{x}^{\text {las }}$ corresponding to the maximally localized Harper-Hofstadter eigenvector, but the results are qualitatively independent of this choice. Fixing $k_{x}^{\text {las }}$ is, however, needed if one is to compute the KPZ correlation functions by running parallel simulations.

[67] D. S. Wiersma, The Physics and Applications of Random Lasers, Nat. Phys. 4, 359 (2008).
[68] F. Baboux, D. D. Bernardis, V. Goblot, V. N. Gladilin, C. Gomez, E. Galopin, L. L. Gratiet, A. Lemaître, I. Sagnes, I. Carusotto, M. Wouters, A. Amo, and J. Bloch, Unstable and Stable Regimes of Polariton Condensation, Optica 5, 1163 (2018).

[69] Certainly, the fact that the edge and bulk $\mathrm{HH}$ modes have very different spatial profiles can be helpful to achieve this goal, as pointed out also in connection to supersymmetric laser arrays [70].

[70] M. Khajavikhan, M. Hokmabadi, J.H. Choi, and D. Christodoulides, Topological and Supersymmetric Laser Arrays (Conference Presentation), in Novel In-Plane Semiconductor Lasers XIX, Vol. 11301, edited by A. A. Belyanin and P. M. Smowton (International Society for Optics and Photonics, Bellingham, WA, 2020).

[71] D. M. Whittaker and P. R. Eastham, Coherence Properties of the Microcavity Polariton Condensate, Europhys. Lett. 87, 27002 (2009).

[72] M. Secli and I. Carusotto, Harper-Hofstadter Topological Laser with Frequency-Dependent Gain, in Proceedings of the

2019 Conference on Lasers and Electro-Optics Europe European Quantum Electronics Conference (CLEO/ Europe-EQEC) (2019), https://ieeexplore.ieee.org/abstract/ document/8872202.

[73] A. Pick, A. Cerjan, and S. G. Johnson, Ab Initio Theory of Quantum Fluctuations and Relaxation Oscillations in Multimode Lasers, J. Opt. Soc. Am. B 36, C22 (2019).

[74] F. Baboux, Kpz... (to be published).

[75] J. Bezanson, A. Edelman, S. Karpinski, and V. B. Shah, Julia: A Fresh Approach to Numerical Computing, SIAM Rev. 59, 65 (2017). 